\title{
Differential propagation of ripples along the proximodistal and septotemporal axes of dorsal CA1 of rats
}

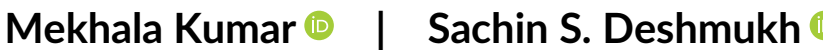

Centre for Neuroscience, Indian Institute of Science, Bangalore, Karnataka, India

\section{Correspondence}

Sachin S. Deshmukh, Centre for Neuroscience, Indian Institute of Science, Bangalore,

Karnataka, India.

Email: sachin@iisc.ac.in

\section{Present address}

Mekhala Kumar, Department of Psychology,

University of Michigan, Ann Arbor, Michigan

Funding information

The Wellcome Trust DBT India Alliance, Grant/ Award Number: IA/S/13/2/501024

\begin{abstract}
The functional connectivity of the hippocampus with its primary cortical input, the entorhinal cortex, is organized topographically. In area CA1 of the hippocampus, this leads to different functional gradients along the proximodistal and septotemporal axes of spatial/sensory responsivity and spatial resolution respectively. CA1 ripples, a network phenomenon, allow us to test whether the hippocampal neural network shows corresponding gradients in functional connectivity along the two axes. We studied the occurrence and propagation of ripples across the entire proximodistal axis along with a comparable spatial range of the septotemporal axis of dorsal CA1. We observed that ripples could occur at any location, and their amplitudes were independent of the tetrode location along the proximodistal and septotemporal axes. When a ripple was detected on a particular tetrode ("reference tetrode"), however, the probability of cooccurrence of ripples and ripple amplitude observed on the other tetrodes decreased as a function of distance from the reference tetrode. This reduction was greater along the proximodistal axis than the septotemporal axis. Furthermore, we found that ripples propagate primarily along the proximodistal axis. Thus, over a spatial scale of $\sim 1.5 \mathrm{~mm}$, the network is anisotropic along the two axes, complementing the topographically organized cortico-hippocampal connections.
\end{abstract}

\section{KEYWORDS}

hippocampus, lateral Entorhinal cortex (LEC), medial Entorhinal cortex (MEC), proximodistal (transverse) axis, ripples, septotemporal (longitudinal) axis

\section{I INTRODUCTION}

The hippocampal proximodistal and septotemporal axes have an interesting connectivity pattern with their input regions. The hippocampus receives its primary cortical input from the entorhinal cortex (EC). The hippocampus and EC have a reciprocal anatomical connectivity showing two orthogonal patterns (Witter \& Amaral, 2004). The medial entorhinal cortex (MEC) projects preferentially to proximal CA1, and the lateral entorhinal cortex (LEC) to distal CA1 (Naber, Lopes da Silva, \& Witter, 2001; Steward \& Scoville, 1976). Dorso-caudal MEC and lateral LEC project to septal hippocampus, while rostro-ventral MEC and medial LEC project to temporal hippocampus (Naber et al., 2001). Connections from CA1 and the subiculum to EC also maintain this topographical organization (Naber et al., 2001; Witter \& Amaral, 2004), creating a loop. In addition to the differential connectivity along the two orthogonal axes of $\mathrm{EC}$, the nature of information sent to the hippocampus by EC also varies along these axes. MEC shows path integration derived spatial representation, while LEC shows sensory derived nonspatial as well as spatial representation (Deshmukh \& Knierim, 2011; Hafting, Fyhn, Molden, Moser, \& Moser, 2005; Hargreaves, Rao, \& Knierim, 2005; Knierim, Neunuebel, \& Deshmukh, 2014). Grid spacing increases in discrete steps along the dorso-ventral axis of MEC (Stensola et al., 2012), though no such functional gradient has been demonstrated yet along the lateromedial axis of LEC. Corresponding to the functional differences in EC, the hippocampus shows functional differences along its two 
orthogonal axes. The proximodistal axis of CA1 shows differences in spatial and sensory encoding (Henriksen et al., 2010; Oliva, Fernández-Ruiz, Buzsáki, \& Berényi, 2016b), while the septotemporal axis shows an increase in the scale of spatial representation (Jung, Wiener, \& McNaughton, 1994; Maurer, VanRhoads, Sutherland, Lipa, \& McNaughton, 2005). In order to better understand the link between the structure and the function of the hippocampus described above, it is essential to understand how information flows through these networks involving the hippocampus.

The sharp-wave ripple complex is one such network phenomenon that may inform us about the hippocampal network organization. Depolarization of the apical dendrites of CA1 pyramidal cells by synchronous bursting of CA3 pyramidal cells causes sharp-waves in CA1. Ripples, high frequency oscillations $(150-250 \mathrm{~Hz})$ caused by the coordinated activation of CA1 pyramidal cells and interneurons, often accompany sharpwaves. These sharp-wave ripple complexes are observed during periods of awake immobility, non-REM sleep, and consummatory behaviors (Buzsáki, 2015; Buzsáki, Horváth, Urioste, Hetke, \& Wise, 1992; Csicsvari, Hirase, Czurkó, Mamiya, \& Buzsáki, 1999; Ylinen et al., 1995).

Along the septotemporal axis, ripples show a reduction in amplitude as a function of distance from any reference electrode, and a tendency to propagate (Patel, Schomburg, Berényi, Fujisawa, \& Buzsáki, 2013). We tested if these patterns hold along the proximodistal axis. We compared these proximodistal patterns with those along a comparable spatial extent along the septotemporal axis in dorsal CA1 in our dataset. We show that there is a decrease in amplitude and probability of cooccurrence as a function of distance from the reference, and that ripples travel preferentially along the proximodistal axis.

\section{2 | METHODS}

\subsection{Animals and surgery}

All animal procedures were performed in accordance with the National Institutes of Health $(\mathrm{NIH})$ animal use guidelines and were approved by the Johns Hopkins University Animal Care and Use Committee. Local field potentials (LFPs) were recorded from eight male, 5-6 month old Long Evans rats. A hyperdrive was implanted over the right dorsal hippocampus (see Deshmukh \& Knierim, 2011 for details of surgical procedures). The hyperdrive had approximately three rows of guide cannulae (15 independently movable tetrodes and 2 references) angled at $35^{\circ}$ to the $\mathrm{ML}$ axis and the lateral/anterior-most tetrode was positioned at $\sim 3.4 \mathrm{~mm}$ posterior and $3.4 \mathrm{~mm}$ lateral to bregma in order to record from the entire proximodistal axis of CA1.

\section{2 | Behavioral protocol}

All rats were trained and tested on a circular track (see Knierim, 2002 for details). Resting sessions were recorded while the rats were made to sit on a pedestal for about 20 minutes before the first session and after the last session on each day. LFPs from only the resting sessions were analyzed and have been presented here. We did not record videos, EEG/cortical LFPs, or EMGs or keep track of the behavioral state of the rats during these resting sessions. Thus, we could not segregate the periods of awake immobility from the periods of sleep.

\subsection{Data acquisition}

Neural recordings were performed using a 64-channel wireless transmitter (Triangle Biosystems, Durham, NC) connected to a Cheetah Data Acquisition system (Neuralynx, Bozeman, MT). LFPs were recorded from one channel from each tetrode after amplifying 2000 -fold, filtering between 1 and $475 \mathrm{~Hz}$, and digitizing at $1 \mathrm{kHz}$.

\section{4 | Histology}

Electrolytic lesions were made on the last day of recording on a few of the tetrodes (typically $2 /$ rat). The recording location of each tetrode was reconstructed from tetrode tracks in coronal sections, and the lesions on the small subset of tetrodes were used for confirmation. For quantitative purposes, relative distances along the proximodistal and septotemporal axes were estimated from histology.

\section{$2.5 \quad$ Ripple detection}

Theta epochs were excluded from the analysis. To detect ripples, the root mean square (RMS) amplitude of LFPs filtered between $150-250 \mathrm{~Hz}$ was calculated using a moving window of $10 \mathrm{~ms}$. A threshold of $2 S D$ above mean was then used to first detect putative ripple events. The start and end time points (boundaries) of these putative events were detected using a threshold of 1 SD above mean, and only the events with durations between $25 \mathrm{~ms}$ and $200 \mathrm{~ms}$ were classified as ripples. In addition to collectively analyzing all ripple events $\geq$ mean $+2 S D$, the ripple events were classified into amplitude groups of mean + 2-4SD, mean + 4-6SD, and $\geq$ mean + $6 S D$ and analyzed separately. These will be referred to as $\geq 2 S D, 2-4 S D, 4-6$ $S D$, and $\geq 6 S D$ through the rest of the manuscript. The time of the peak amplitude of a ripple obtained from the envelope of the analytical signal was used as the time of occurrence of the ripple. After detecting ripple events, ripple bursts (ripple doublets, triplets, etc.) on any given tetrode were detected as those events that had a time difference in occurrence between 40 and 200 ms.

In the analyses that follow, each tetrode served as a reference against which every other tetrode was compared for comparing ripple properties at different relative anatomical distances.

\section{6 | Data considered for analysis}

LFP recordings from only the tetrodes in the pyramidal cell layer of CA1 showing ripples were included (Figure 1). For each rat, a total of 
(a) Histological sections of tetrode locations from one rat

103

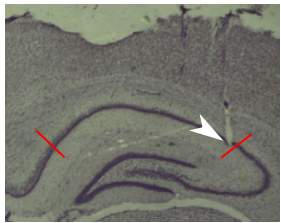

$\mathrm{PD}$ distance $=0 \mathrm{~mm}$

ST distance $=0.23 \mathrm{~mm}$

118

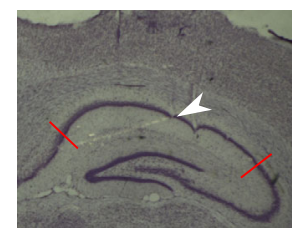

PD distance $=0.8 \mathrm{~mm}$ ST distance $=0.53 \mathrm{~mm}$

121

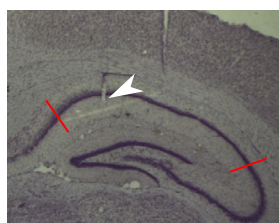

PD distance $=1.39 \mathrm{~mm}$ ST distance $=0.31 \mathrm{~mm}$
110

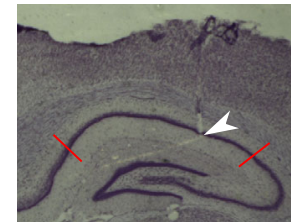

PD distance $=0.39 \mathrm{~mm}$ ST distance $=0.36 \mathrm{~mm}$

114

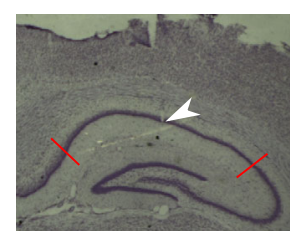

PD distance $=0.85 \mathrm{~mm}$ ST distance $=0.27 \mathrm{~mm}$

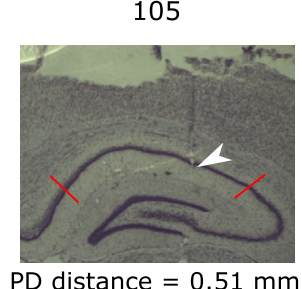

ST distance $=0 \mathrm{~mm}$

132

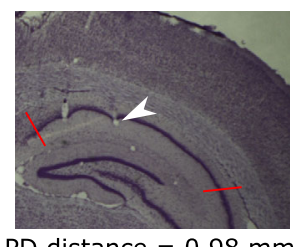

PD distance $=0.98 \mathrm{~mm}$ ST distance $=1.2 \mathrm{~mm}$ (b) Rat-wise tetrode positions

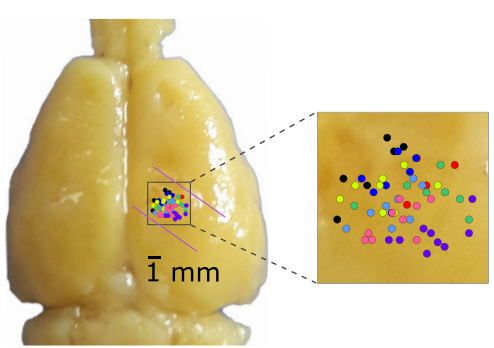

(c)

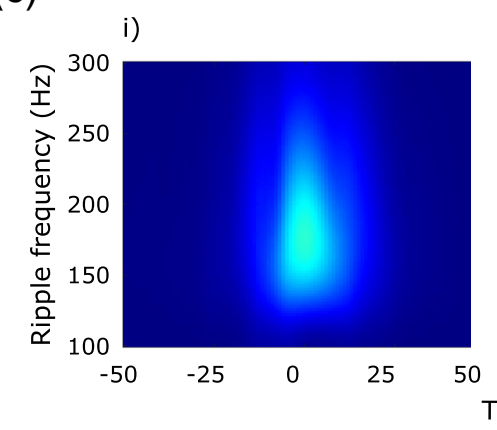

130

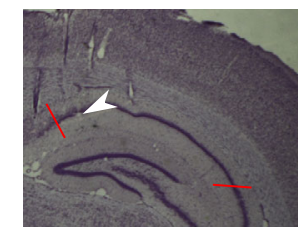

PD distance $=1.58 \mathrm{~mm} \quad P D$ distance $=1.71 \mathrm{~mm}$
131

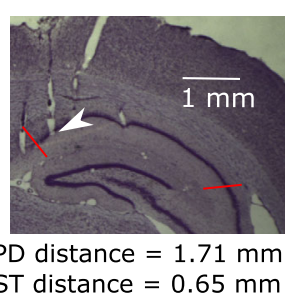

Average wavelet spectrograms

ii)

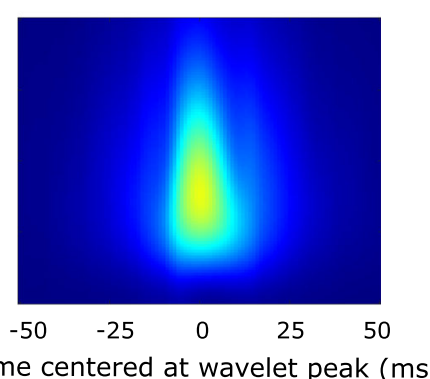

iii)

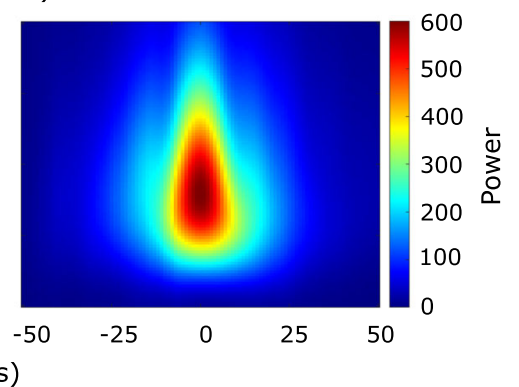

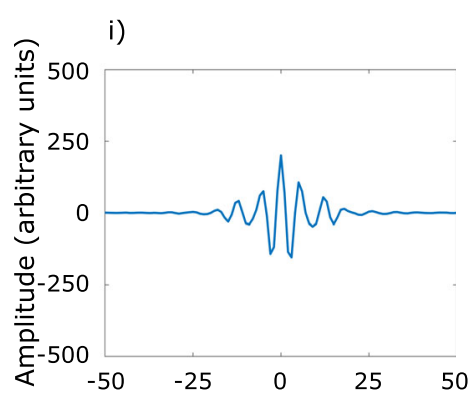

ii) Average ripple traces

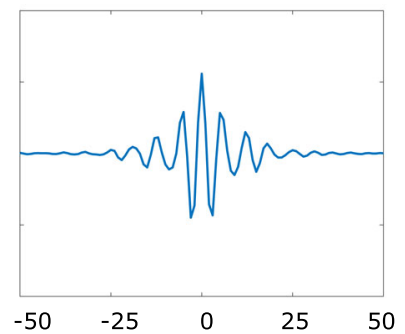

Time centered at ripple peak (ms) iii)

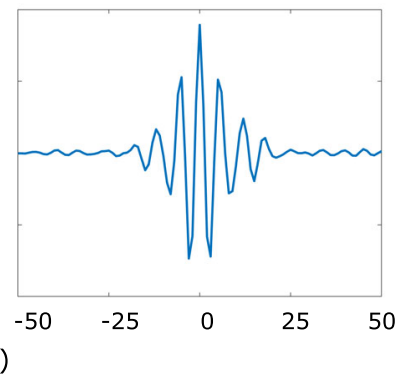

FIGURE 1 Legend on next page. 
$4-8$ resting sessions were pooled. The rate of $\geq 2$ SD ripple occurrence across sessions and rats was $47 \pm 10 \mathrm{ripples} / \mathrm{min}$. In all, the dataset included 305,548 ripples from 58 tetrodes in 38 sessions in 8 rats.

\section{7 | Detection of cooccurring ripples}

A ripple on a referred tetrode was considered to cooccur with a ripple on a reference tetrode if there was an overlap between the boundaries of the two ripples. To reduce the likelihood of a ripple cooccurring on the referred tetrode going undetected due to a reduction in amplitude, the threshold for ripple detection on the referred tetrode was maintained at $2 S D$ above mean (other criteria remained unchanged) irrespective of the threshold on the reference tetrode. For each reference-referred pair, the fraction of ripples on the reference tetrode having cooccurring ripples on the referred tetrode was computed.

\section{8 | Measurement of relative amplitude}

The amplitude of a ripple was measured as the peak with the largest amplitude in an identified ripple. If the signal on the referred tetrode was below the ripple detection threshold, the peak amplitude on the referred tetrode was measured within the boundaries of the ripple on the reference tetrode. A variety of factors, including exact location of the tetrode in the pyramidal cell layer (Mizuseki, Diba, Pastalkova, \& Buzsáki, 2011; Ylinen et al., 1995) as well as tetrode impedance affect the recorded ripple amplitude. To account for tetrode to tetrode variability, amplitudes measured on each tetrode were normalized by the median ripple amplitude on that tetrode. The ratio of the normalized amplitude on the referred tetrode to the normalized amplitude on the reference tetrode was calculated as the relative amplitude. For each reference-referred pair, the mean of all the relative amplitudes was computed.

\section{9 | Measurement of ripple frequency}

Wavelet transform was used to compute the frequency of ripples. Ripple frequency was measured as the frequency bin in which the wavelet transform had the maximum power.

\subsection{0 | Propagation of ripples}

In order to understand ripple propagation, each of the four extreme tetrodes (the proximal-most, distal-most, septal-most, and temporalmost tetrodes) in each of the hyperdrives were used as reference tetrodes to detect ripples. Ripples were then sequentially detected on the referred tetrodes in order of increasing distance from the reference tetrode overlapping with the cooccurring ripple on the preceding tetrode to identify a putative propagating event. Any common events detected between the four extreme reference tetrodes were counted only once. Only those events having at least six consecutive tetrodes with ripples and spanning a minimum distance of $0.6 \mathrm{~mm}$ along the proximodistal and septotemporal axes were analyzed further (rat 305 had only 4 tetrodes and was, therefore, included only in the preceding analyses of ripple rate, amplitude, cooccurrence, etc., but excluded from the ripple propagation analysis). Multiple linear regression was then performed on the time of occurrence of ripples vs. relative distance along the proximodistal and septotemporal axes. An event was considered as propagating if the fit was significant (at $p<$ $0.05)$. Some events were common between the 2-4 SD, 4-6 SD, and $\geq 6 S D$ amplitude groups, for example, if an event having a ripple falling in the amplitude group of $\geq 6 S D$ on one extreme tetrode had a ripple falling in the amplitude group of 2-4 SD on another extreme tetrode, the event would be detected for $2-4 S D$ as well as $\geq 6 S D$. These events were counted only once by including the events only in the highest amplitude group that they belonged to.

To find the direction of propagation of each of these events, vector algebra was employed. The partial slopes along the two orthogonal axes viz. the proximodistal and septotemporal axes obtained from the multiple linear regression analysis were considered as component vectors, their magnitude defined by the absolute value of the partial slope and their direction along their respective axis defined by the sign (+/-) of the partial slope. The resultant of the two orthogonal component vectors was then computed to obtain the resultant slope and angle of propagation, which was calculated with respect to the proximodistal direction. Thus, propagation along the proximodistal and distoproximal directions were defined by the angles $0^{\circ}$, and $180^{\circ}$ or $-180^{\circ}$ respectively, while propagation along the septotemporal and temporoseptal directions were defined by the angles $90^{\circ}$ and - $90^{\circ}$ respectively. Intermediate directions were defined by intermediate angles.

The average speed along each of the four directions was measured by first categorizing events into the four directions of

FIGURE 1 Tetrode positions and averaged ripple traces. (a) Histological sections showing tetrode locations from one rat (rat 441). Arrows indicate tetrodes included in the analysis. Other tracks belong to tetrodes excluded from the analysis since they were not in the CA1 pyramidal cell layer on the days included in the analysis. Numbers atop each section indicate the section number. Sections were $47 \mu \mathrm{m}$ thick after accounting for $15 \%$ histological shrinkage factor. (b) Distribution of all tetrodes used for the analysis across the proximodistal and septotemporal axes of CA1. Dots of a color represent tetrodes from a single rat. Relative tetrode positions obtained from histology were marked on the whole brain for display purposes using the estimates obtained from the rat brain atlas (Paxinos \& Watson, 2007). Purple lines indicate the orientation of the septotemporal axis. (c) Averaged wavelet spectrograms (top) and ripple traces (bottom) for 2-4 SD (i), 4-6 SD (ii), and $\geq 6$ SD (iii) from one randomly chosen tetrode from a randomly chosen rat (rat 302) [Color figure can be viewed at wileyonlinelibrary.com] 
propagation using bin widths of $\pm 45^{\circ}$ about the expected angle of each of the four directions. For each direction, the relative time of occurrence of all ripples on each tetrode with respect to the ripples on the tetrode with the earliest time of occurrence was averaged across events. Multiple linear regression was performed on each of the four averaged datasets, and the average speed for each of the four directions was computed as the inverse of the resultant slope. Additionally, the speed was separately calculated for each propagating event as the inverse of the resultant slope for each event.

To ensure that propagation in the observed preferred direction and speed did not occur by chance, a shuffle analysis was performed. For each propagating event, the time of occurrence of each of the ripples was randomly permuted with respect to the tetrode positions to generate the shuffled dataset. The multiple linear regression analysis and significance criteria were then applied to each of the shuffled events as was done for the observed data. This entire process was done iteratively to generate 1,000 such datasets; for a given rat, for every iteration a single random permutation of the time of occurrence with respect to tetrode positions was used for all propagating events. To quantify the possible differences in the preferred direction of propagation (proximodistal vs. septotemporal) between the observed data and shuffle data, we calculated the ratio of the number of events propagating along the proximodistal and distoproximal directions to those along the septotemporal and temporoseptal directions: slopes along the proximodistal and septotemporal axes. Two-tailed test of proportions was used to compare proportions of propagating ripples in the different directions. Two-tailed Wilcoxon signed-rank test (MATLAB function signrank) was used to compare proportions of events classified into the four directions of propagation having longduration ripples (> $100 \mathrm{~ms}$ ) and ripple bursts for propagating and nonpropagating ripples.

\section{3 | RESULTS}

We recorded LFPs across the entire proximodistal axis and a comparable spatial range along the septotemporal axis from dorsal CA1 of rats (Figure 1, Supplementary Figure 1) during resting sessions. Ripples were detected using a threshold of $2 S D$ above mean. All ripples $\geq 2$ $S D$ were collectively analyzed, and additionally, were classified into 2-4 SD, 4-6 SD, and $\geq 6$ SD and analyzed separately. To look for possible differences in the occurrence of ripples, we first examined whether the rate of ripple occurrence was influenced by the positions of the tetrodes along the proximodistal and septotemporal axes. We observed no differences in the rates for $\geq 2 S D$ (Figure 2a). However, the rate was higher in proximal CA1 than distal CA1 for 2-4 SD (Figure 2b), did not show a significant trend for 4-6SD(Figure 2c), and was higher in distal CA1 than proximal CA1 for $\geq 6$ SD (Figure 2d).

ratio for direction $=\frac{\text { no.of events }\left(-45^{\circ} \leq \operatorname{dir}<45^{\circ}\right)+\text { no. of events }\left(-180^{\circ} \leq \operatorname{dir}<-135^{\circ}\right)+\text { no.of events }\left(135^{\circ}<\operatorname{dir} \leq 180^{\circ}\right)}{\text { no.of events }\left(-135^{\circ} \leq \operatorname{dir}<-45^{\circ}\right)+\text { no.of events }\left(45^{\circ}<\operatorname{dir} \leq 135^{\circ}\right)}$

where "dir" denotes direction. In addition to this, for the observed data, the distribution of the partial slopes along the proximodistal axis was bimodal but that along the septotemporal axis was not. Therefore, we calculated the following to quantify possible differences in the distribution of the partial slopes of the observed and shuffled data:

ratio for slopes $=\frac{\text { no.of events }(-1 \leq \text { proximo }- \text { distal partial slope } \leq 1)}{\text { no.of events }(-1 \leq \text { septo }- \text { temporal partial slope } \leq 1)}$

A range of $\pm 1 \mathrm{~ms} / \mathrm{mm}$ was used as the separation in the two populations along the proximodistal axis was evident within this range for the observed data. The observed data was considered to be significantly different from chance if less than $1 \%$ of the 1,000 shuffled iterations had ratios for direction more than that of the observed data and ratios for slopes less than that of the observed data.

\subsection{Statistical analysis}

All statistics used a significance criterion of $p<0.05$ unless mentioned otherwise. One-tailedt-tests were used to compare partial
There was no such gradient along the septotemporal axis for any of the three subgroups (Figure $2 b-d$ ). After controlling for differences in the spatial spreads of tetrodes across the proximodistal and septotemporal axes, there were no significant differences in ripple rates as a function of tetrode position (Supplementary Figures 2 and 3). We also looked at properties such as ripple amplitude, duration, frequency, and ripple bursts. Ripple amplitude and frequency showed no differences across the proximodistal and septotemporal axes for any of the amplitude groups (Supplementary Figures 3 and 5; Supplementary Tables 4 and 6). We also observed no differences in ripple duration across the two axes for $\geq 2 S D$ and 2-4 SD. Ripple duration was significantly higher in proximal CA1 than distal CA1 for 4-6 SD and $\geq 6 S D$ but showed no difference across the septotemporal axis (Supplementary Figures 4 and 5). The proportions of long-duration ripples (> $100 \mathrm{~ms}$ ) and ripple bursts also showed no differences across the two axes (Supplementary Figure 6). In summary, only ripple duration and rate of ripple occurrence showed variation along the proximodistal but not the septotemporal axis in specific amplitude groups.

Next, we asked whether these ripples cooccurred with each other (Figure 3). For each reference tetrode, we looked at the fraction of 
(a)

i) Three dimensional view

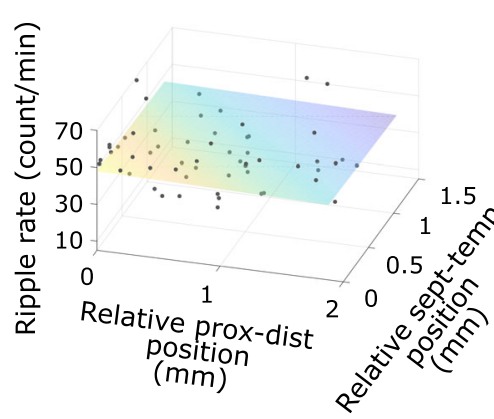

(b)

i) Three dimensional view

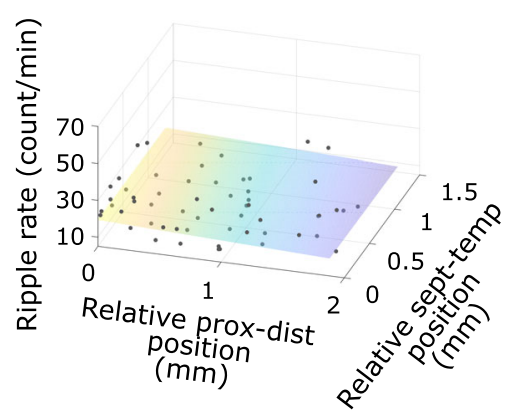

(c)

i) Three dimensional view

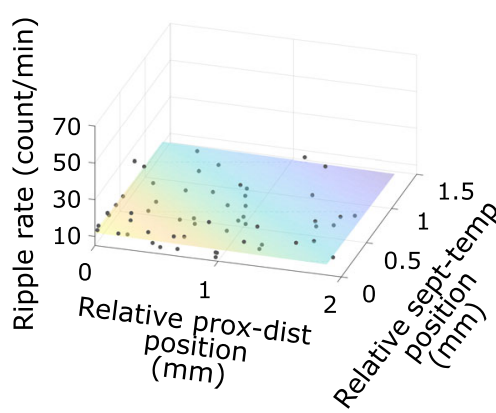

(d)

i) Three dimensional view

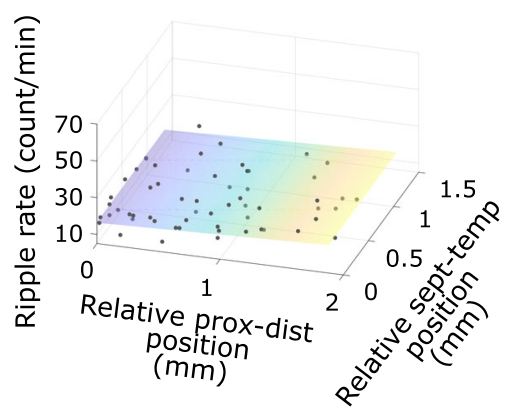

$\geq 2$ SD

ii) Proximo-distal projection

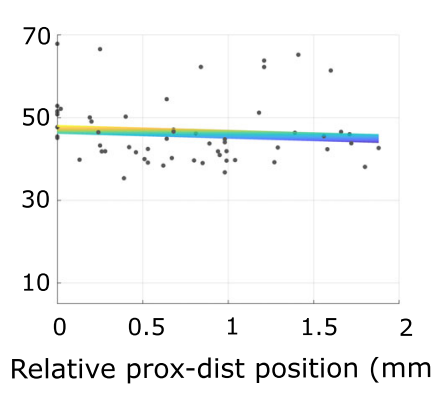

iii) Septo-temporal projection

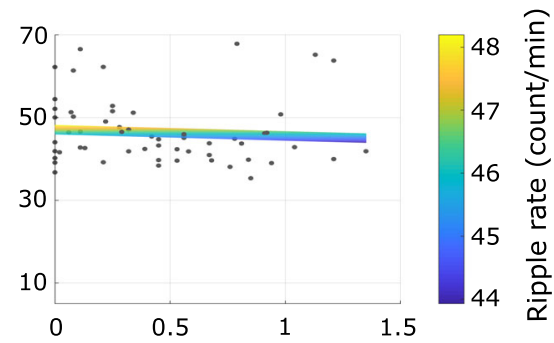

Relative sept-temp position $(\mathrm{mm})$
2-4 SD

ii) Proximo-distal projection iii) Septo-temporal projection
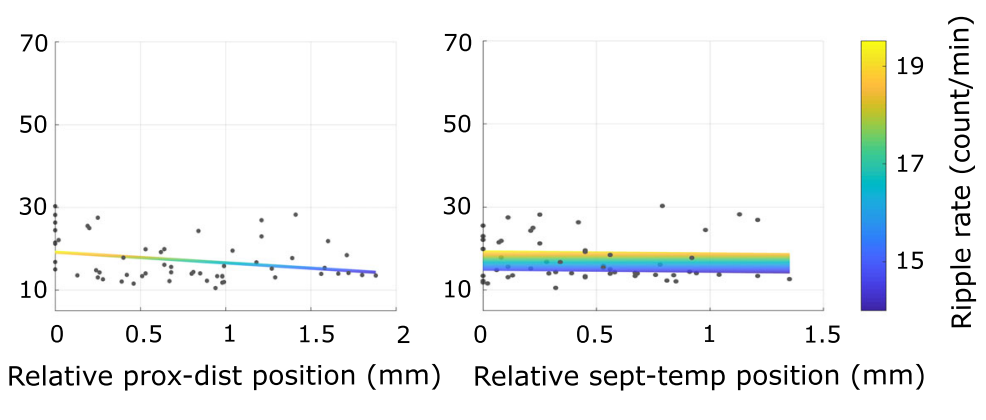

Relative sept-temp position $(\mathrm{mm})$
4-6 SD

ii) Proximo-distal projection

iii) Septo-temporal projection
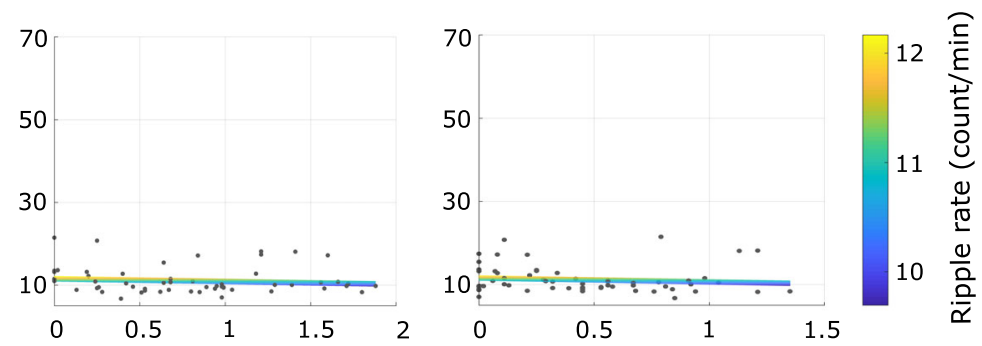

Relative prox-dist position ( $\mathrm{mm}$ ) Relative sept-temp position ( $\mathrm{mm}$ ) $\geq 6 \mathrm{SD}$

ii) Proximo-distal projection

iii) Septo-temporal projection

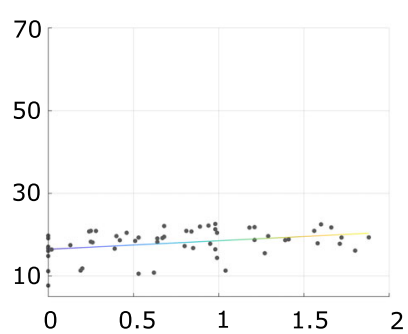

Relative prox-dist position ( $\mathrm{mm}$ ) Relative sept-temp position ( $\mathrm{mm}$ )

FIGURE 2 Legend on next page. 


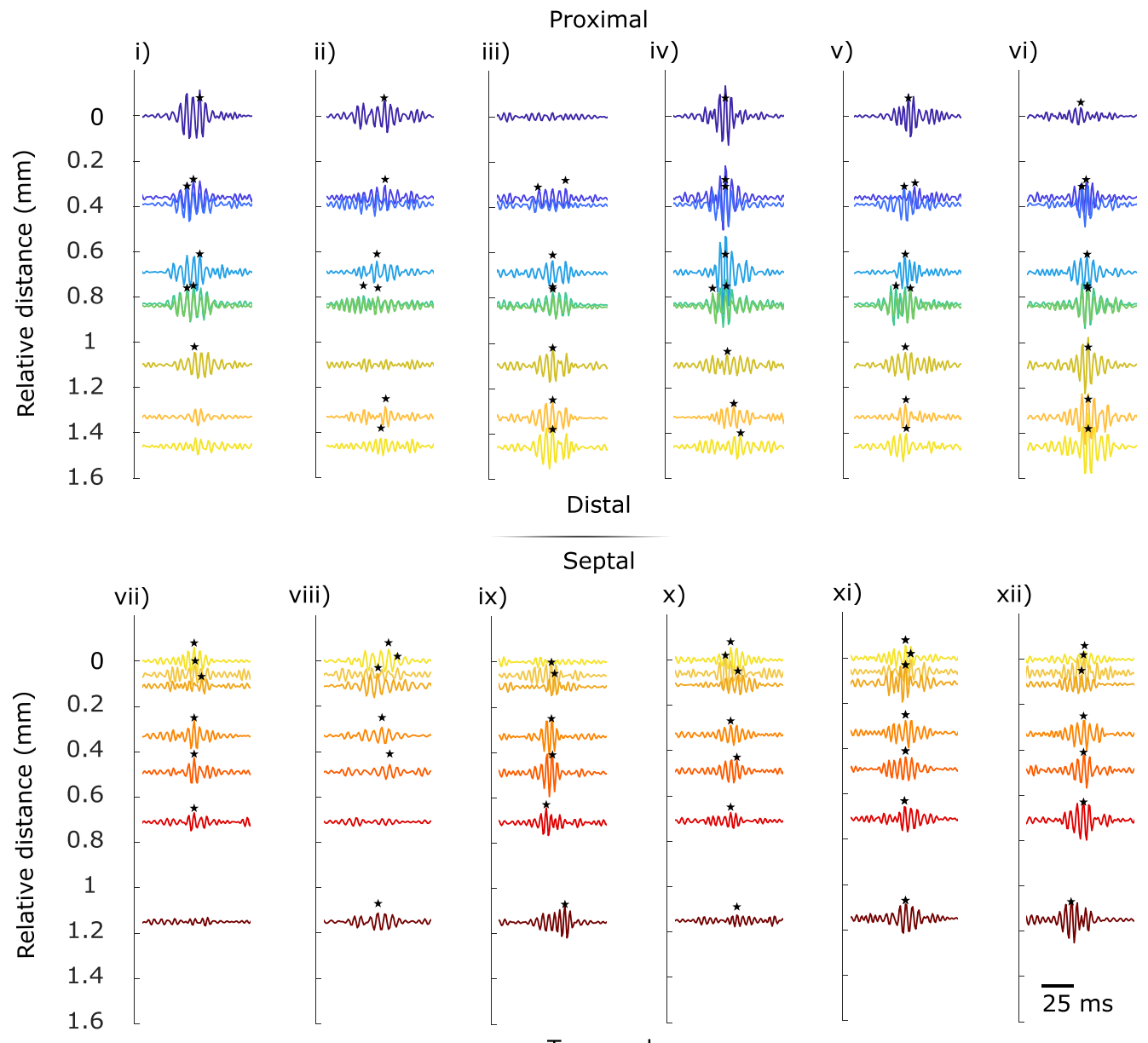

FIGURE 3 Examples of ripple patterns across tetrodes. Recorded traces are arranged from proximal to distal in (i)-(vi) (rat 432) while traces are arranged from septal to temporal in (vii)-(xii) (rat 392). Stars indicate detected ripples on a tetrode and are positioned at the time of peak amplitude of the ripples. (i)-(iii) and (vii)-(ix) show absence of cooccurring ripples on some of the tetrodes. (iv)-(vi) and (x)-(xii) show ripples of different amplitudes cooccurring on all tetrodes [Color figure can be viewed at wileyonlinelibrary.com]

ripples having cooccurring ripples on all the referred tetrodes. We quantified the correlation between cooccurrence and distance by plotting the fraction of cooccurring ripples as a function of the absolute distance between all pairs of tetrodes along both axes (Figure 4). The likelihood of cooccurrence decreased with increasing distance for $\geq 2 S D$ (Figure 4a). This decrease was significant along the proximodistal axis but not the septotemporal axis and was significantly steeper along the proximodistal axis than the septotemporal axis. Consistent with this, the fraction of cooccurring ripples decreased with increasing distance for 2-4 SD, 4-6 SD, and $\geq 6 S D$ (Figure $4 \mathrm{~b}-\mathrm{d}$ ). Notice that the reference ripples in the lower amplitude group were less likely to have cooccurring ripples even for short distances between tetrode pairs, while reference ripples in the higher amplitude group were much more likely to have cooccurring ripples. The decrease in the fraction of cooccurring ripples was significant along the proximodistal axis for all three subgroups. The decrease was not significant along the septotemporal axis for 2-4 SD, but was significant for 4-6 SD and $\geq 6 S D$. The rate of decrease in the fraction of cooccurring ripples was significantly higher along the proximodistal axis than along the septotemporal axis. The results remained similar after controlling for the direction from the reference to the referred tetrode along the two axes (e.g., by segregating comparisons between

FIGURE 2 Rate of ripple occurrence. Rate of ripple occurrence as a function of position along the proximodistal and septotemporal axes for all rats collectively with the $2 D$ fit (plane) for $\geq 2 S D$ (a), 2-4 SD (b), 4-6 $S D$ (c), and $\geq 6 S D$ (d). Column (i) shows a 3D (X-Y-Z) view, (ii) shows a proximodistal projection (X-Z view), and (iii) shows a septotemporal projection ( $\mathrm{Y}-\mathrm{Z}$ view) of column (i). Note the decreasing trend in (b) and the increasing trend in (d) along the proximodistal axis. (a) Proximodistal partial slope $=-1.2 \mathrm{count} / \mathrm{min} / \mathrm{mm}, p=0.54$; septotemporal partial slope $=-1.5 \mathrm{count} / \mathrm{min} / \mathrm{mm}, p=0.59$; one-tailedt-test for comparison of slopes along the two axes, $p=0.53$. See Supplementary Table 1 for statistics after subdividing ripples by amplitude and Supplementary Table 2 for ripple rate as a function of tetrode position in individual rats [Color figure can be viewed at wileyonlinelibrary.com] 

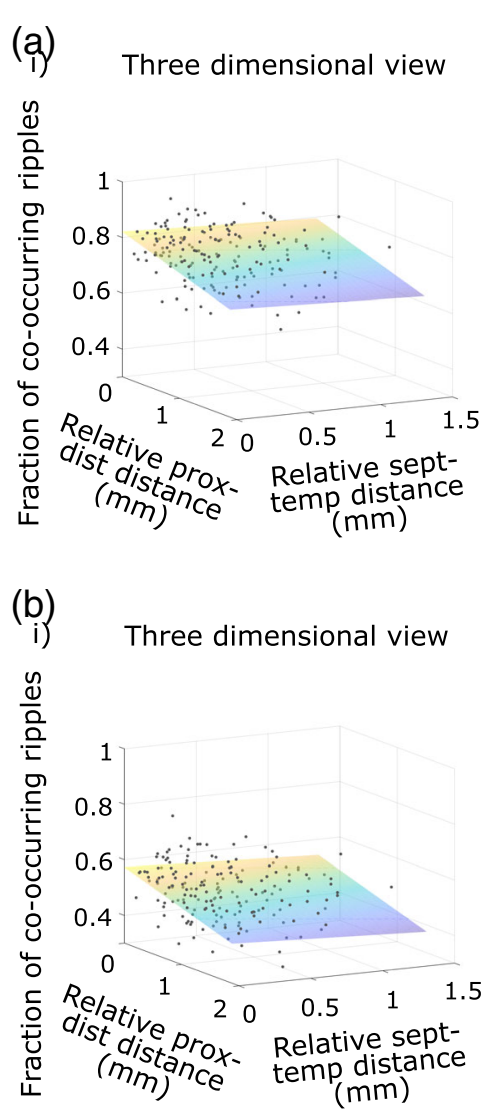

(c)

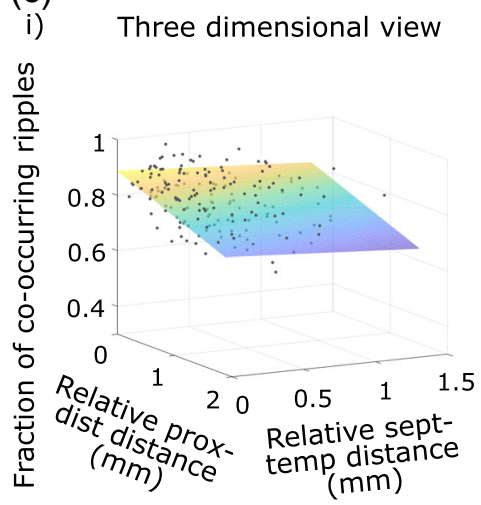

(d)

i) Three dimensional view

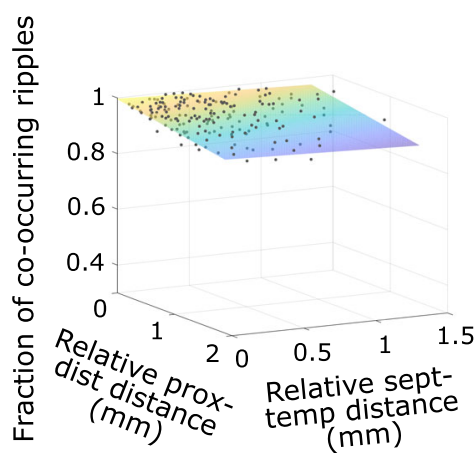

FIGURE 4 Legend on next page. $\geq 2 \mathrm{SD}$

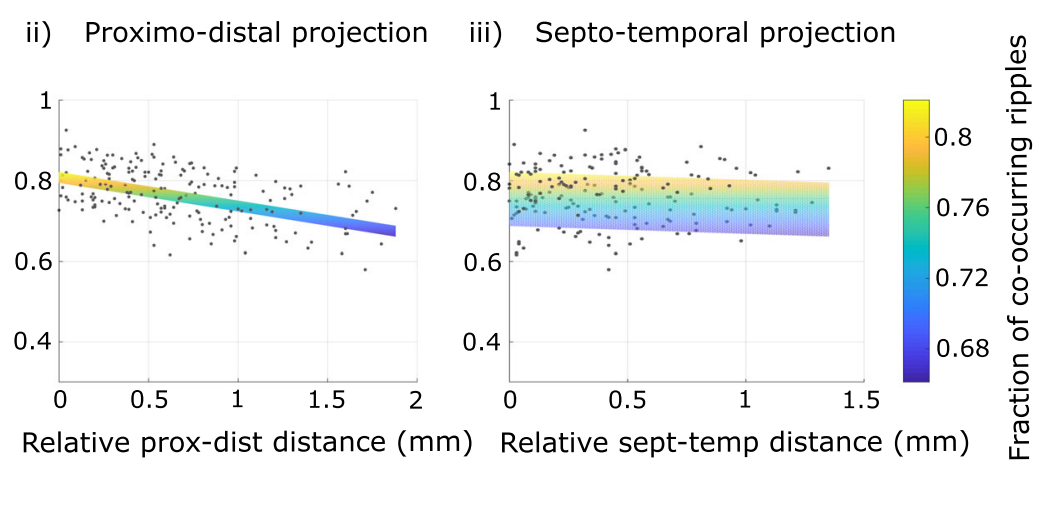

2-4 SD

ii) Proximo-distal projection iii) Septo-temporal projection
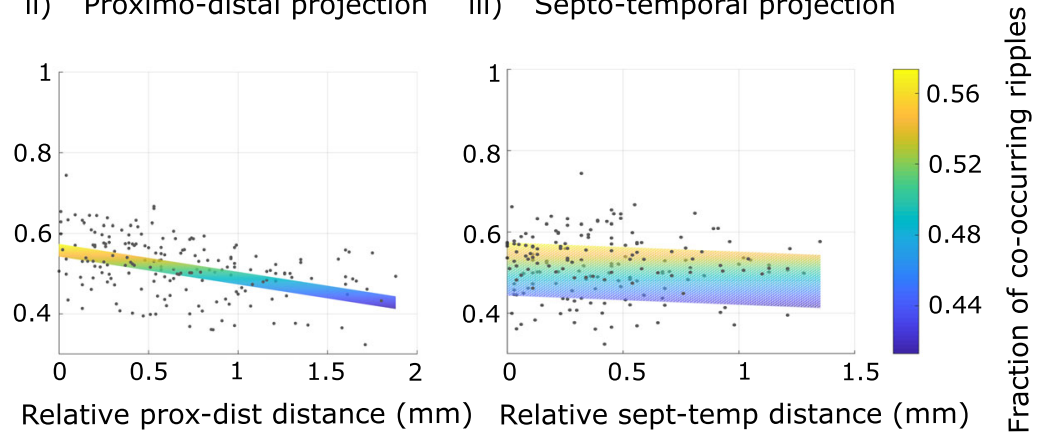

Relative prox-dist distance (mm) Relative sept-temp distance (mm)

\section{4-6 SD}

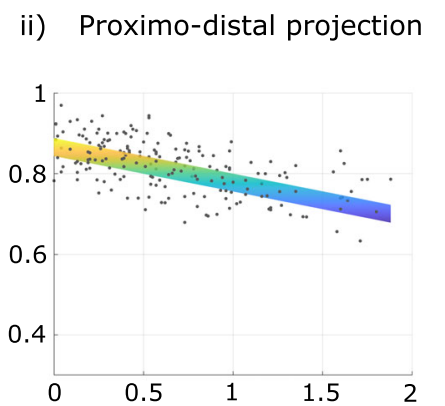

iii) Septo-temporal projection

Relative prox-dist distance $(\mathrm{mm})$ Relative sept-temp distance $(\mathrm{mm})$

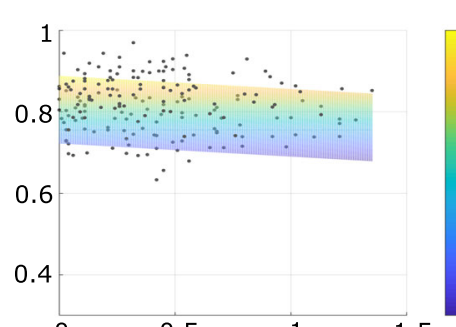

ii) Proximo-distal projection iii) Septo-temporal projection
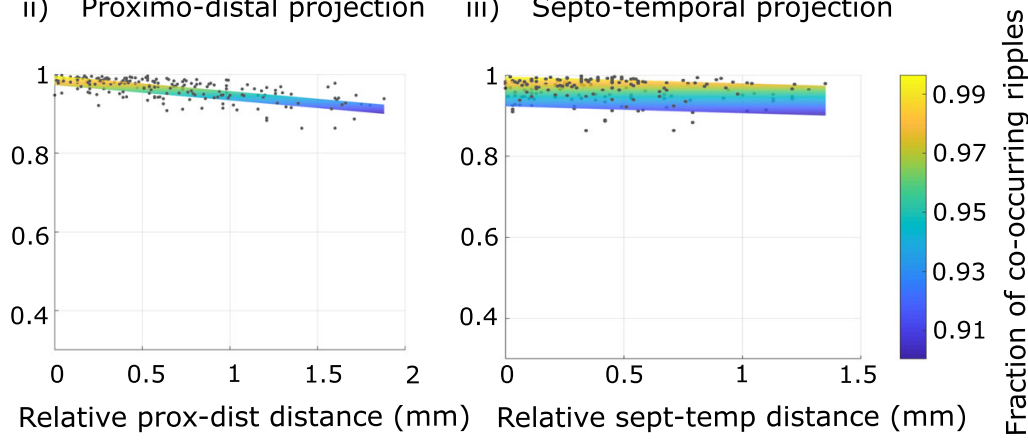

Relative prox-dist distance $(\mathrm{mm})$ Relative sept-temp distance $(\mathrm{mm})$ 造 
pairs having more proximal and septal reference tetrodes than their corresponding referred tetrodes from pairs having more distal and temporal reference tetrodes than their referred tetrodes; Supplementary Figures 7 and 9) and differences in the spatial spreads of tetrodes across the proximodistal and septotemporal axes (Supplementary Figures 8 and 10). Taken together, these results show that the fraction of cooccurring ripples decreased as a function of distance between tetrodes pairs reliably along the proximodistal axis. The decrease along the septotemporal axis was not as reliable.

The decrease in cooccurrence with increasing distance suggested that there may be an amplitude gradient as a function of distance. To test the dependence of ripple amplitude on distance, we measured the relative amplitude of ripples on the referred tetrodes with respect to all ripples on the reference tetrode, irrespective of whether a cooccurring ripple meeting our criterion (mean $+2 S D$ ) was detected on the referred tetrode. As expected, the relative amplitude of ripples decreased with increasing (absolute) distance for $\geq 2 S D$; the decrease was significant along the proximodistal axis but not the septotemporal axis (Figure 5a), with the decrease being significantly steeper along the proximodistal axis than the septotemporal axis. For 2-4 SD and 4-6SD, there was neither an increase nor a decrease along either axis (Figure $5 b, c$ ), but $\geq 6 S D$ showed a significant decrease in relative amplitude along both axes with the decrease being steeper along the proximodistal axis (Figure $5 \mathrm{~d}$ ). The results remained similar after controlling for the direction from the reference to the referred tetrode along the two axes, except the decrease was no longer significant for $\geq 2$ SD (Supplementary Figure 9 and Supplementary Table 13). The results also remained similar after controlling for the differences in the spatial spreads of tetrodes across the proximodistal and septotemporal axes, except the decrease was no longer significant for $\geq 2 S D$ and no longer significantly steeper along the proximodistal axis than the septotemporal axis for $\geq 6$ SD (Supplementary Figure 10 and Supplementary Table 14). Thus, relative ripple amplitude decreased as a function of distance between tetrode pairs reliably along the proximodistal axis for $\geq 6 S D$ ripples on the reference tetrode. The decrease along the septotemporal axis was not as reliable.

Ripples have been shown to propagate over a few $\mathrm{mm}$ along the septotemporal axis (Patel et al., 2013). We tested if ripples propagate similarly along the proximodistal axis, as well as a matching spatial scale $(\sim 1.5 \mathrm{~mm})$ along the septotemporal axis (Figure 6). For $\geq 2 S D$, out of the 25,282 putative propagating events analyzed, 5,199 events (20\%) showed significant propagation (at $p<0.05$ for 2D fit). The distribution of the angles of propagation (with respect to the proximodistal axis) of these events demonstrated a clear preference around $0^{\circ}\left(1,725\right.$ events within $\pm 45^{\circ}$ out of a total of 5,199 propagating events, $33 \%)$ and $180^{\circ}$ and $-180^{\circ}$ (1,559 events, 30\%) over propagation around $-90^{\circ}$ (972 events, $19 \%$ ) and $90^{\circ}$ (943 events, $18 \%$ ) (Figure 7a; ratio for direction $=1.76$; test of proportions comparing the observed proportion of events with the $50 \%$ propagation along the proximodistal axis expected by chance, $Z=20, p<10^{-5 *}$ ) implying that the proximodistal axis was the preferred axis of propagation. Additionally, the distribution of the proximodistal partial slopes about $0 \mathrm{~ms} / \mathrm{mm}$ was bimodal, unlike the septotemporal partial slopes; there were a smaller number of events having proximodistal partial slopes between $\pm 1 \mathrm{~ms} / \mathrm{mm}$ as opposed to the greater number of events with septotemporal partial slopes between $\pm 1 \mathrm{~ms} / \mathrm{mm}$ (ratio $=0.346$; Figure $7 \mathrm{~b}$ ). A slope close to $0 \mathrm{~ms} / \mathrm{mm}$ corresponds to near simultaneous occurrence, and hence a lack of propagation along the given axis. In order to ensure that these results were not an outcome of the criteria used for classifying the events as propagating, we ran a shuffle analysis. The distributions of the angles of propagation for the shuffled data were significantly different from that of the observed data (ratio for direction for the observed data, 1.76, was higher than that for 994 shuffles, $p=0.006^{*}$ ), and so were the distributions of the partial slopes (ratio for slopes for the observed data, 0.346 , was lower than 994 shuffles, $p=0.006 *$ ), thus indicating that the observed result was not by chance. Subdividing putative propagating events by amplitude on the reference tetrode revealed that the bias for propagation along the proximodistal axis was higher for subgroups with higher amplitude on the reference tetrode (Figure 7c; Supplementary Figures 11-13; Supplementary Table 15).

It is possible that events preferentially propagating along the proximodistal axis - events having relatively large, significant proximodistal partial slopes - also had relatively small but significant septotemporal slopes and vice versa for those propagating along the septotemporal axis. Most of the events preferentially propagating in the proximodistal direction showed high $p$-values for septotemporal partial slopes, indicating that these events did not propagate significantly along the septotemporal axis. Similarly, events preferentially propagating along the septotemporal axis showed high $p$-values for proximodistal partial slopes (Figure 7d, Supplementary Figures 11-13), indicating that these events did not propagate significantly along the proximodistal axis. For $\geq 2 S D, 4-6 S D$, and $\geq 6 S D$, notice the substantially higher density of low $p$-values along the proximodistal axis than that along the septotemporal axis. Furthermore, the distribution of the $p$-values of the multiple regression models for all events also showed a greater number of events with $p$ values close to 0 along the proximodistal axis than that along the septotemporal axis (Figure 7e, Supplementary Figures 12 and 13). The distribution of propagation speeds of individual events as a function

FIGURE 4 Ripple cooccurrence. Fraction of cooccurring ripples as a function of relative distance along the proximodistal and septotemporal axes for all rats collectively with the 2D fit (plane) for $\geq 2 S D$ (a), 2-4 SD (b), 4-6 SD (c), and $\geq 6 S D$ (d) ripples on reference tetrodes. Column (i) shows a 3D (X-Y-Z) view, (ii) shows a proximodistal projection (X-Z view), and (iii) shows a septotemporal projection (Y-Z view) of column (i). (a) Proximodistal partial slope $=-0.071$ fraction of cooccurring ripples $/ \mathrm{mm}, p<10^{-5 *}$; septotemporal partial slope $=-0.019$ fraction of cooccurring ripples $/ \mathrm{mm}, p=0.14$; one-tailedt-test for comparison of slopes along the two axes, $p=4 \times 10^{-4 *}$. See Supplementary Table 7 for statistics after subdividing ripples by amplitude on the reference tetrode and Supplementary Table 8 for ripple cooccurrence as a function of relative distance in individual rats [Color figure can be viewed at wileyonlinelibrary.com] 
(a)

i) Three dimensional view

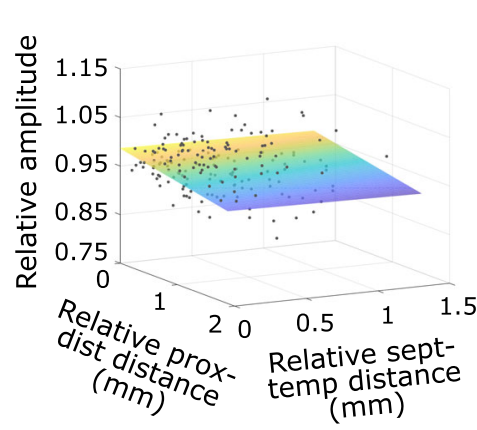

(b)

i) Three dimensional view

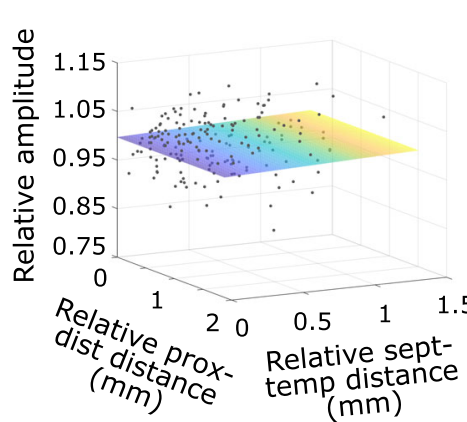

(c)

i) Three dimensional view

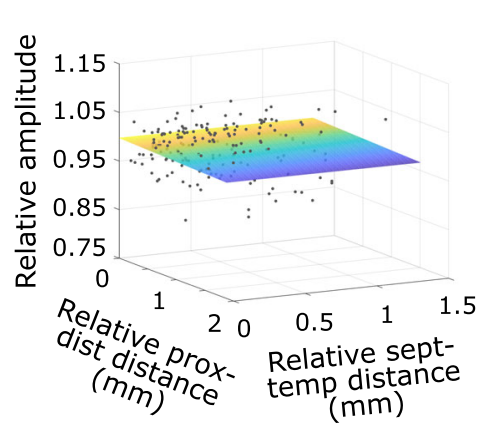

(d)

i) Three dimensional view

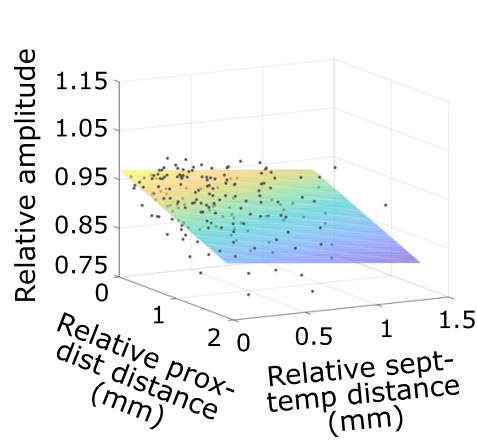
$(\mathrm{mm})$ iii) Septo-temporal projection $\geq 2 \mathrm{SD}$

ii) Proximo-distal projection
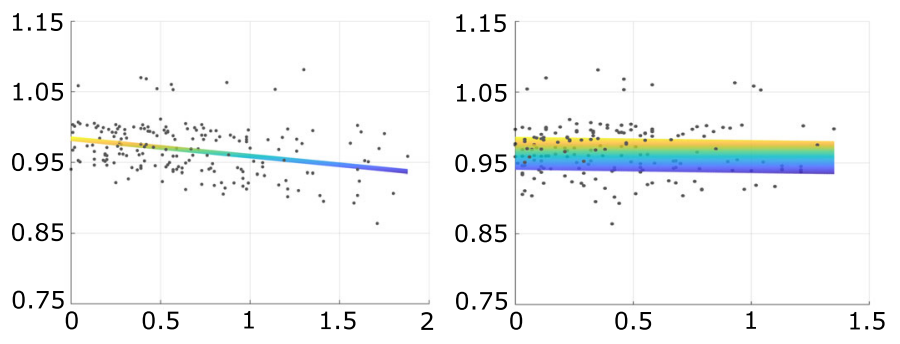

Relative prox-dist distance $(\mathrm{mm})$ Relative sept-temp distance $(\mathrm{mm})$
$0.98 \stackrel{0}{\frac{\pi}{2}}$ 0.97 $0.96 \frac{\varepsilon}{\pi}$ $0.95 \stackrel{2}{\geq}$ $0.94 \frac{10}{2}$
2-4 SD

ii) Proximo-distal projection

iii) Septo-temporal projection

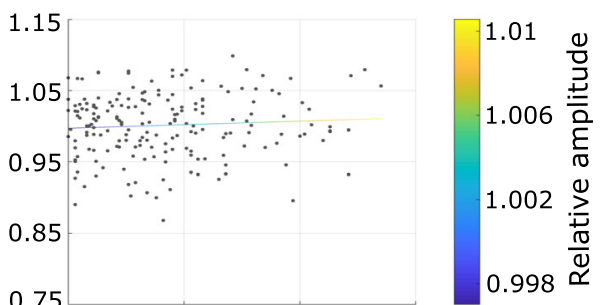

$\begin{array}{lllll}0.75 & & & & \\ 0 & 0.5 & 1 & 1.5 & 2\end{array}$

Relative prox-dist distance $(\mathrm{mm})$ Relative sept-temp distance $(\mathrm{mm})$

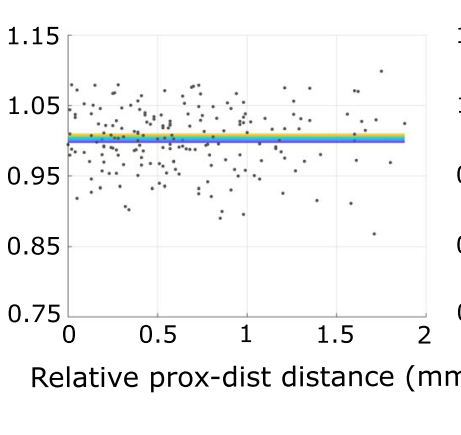

4-6 SD

ii) Proximo-distal projection iii) Septo-temporal projection
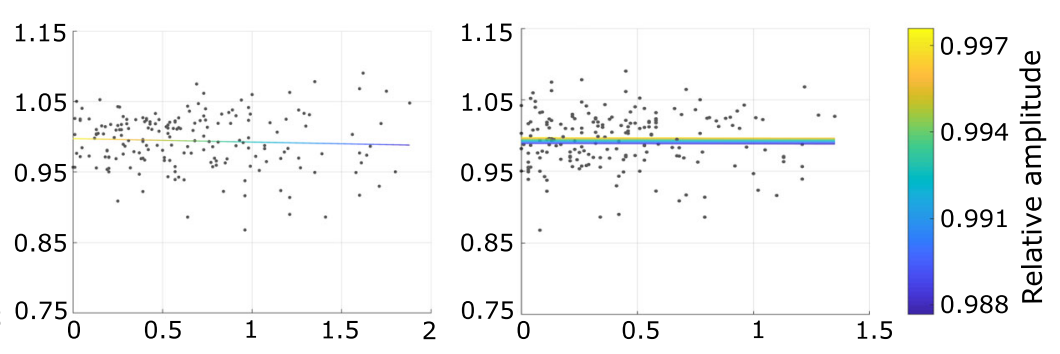

Relative prox-dist distance $(\mathrm{mm})$ Relative sept-temp distance $(\mathrm{mm})$

$\geq 6 \mathrm{SD}$

ii) Proximo-distal projection iii) Septo-temporal projection
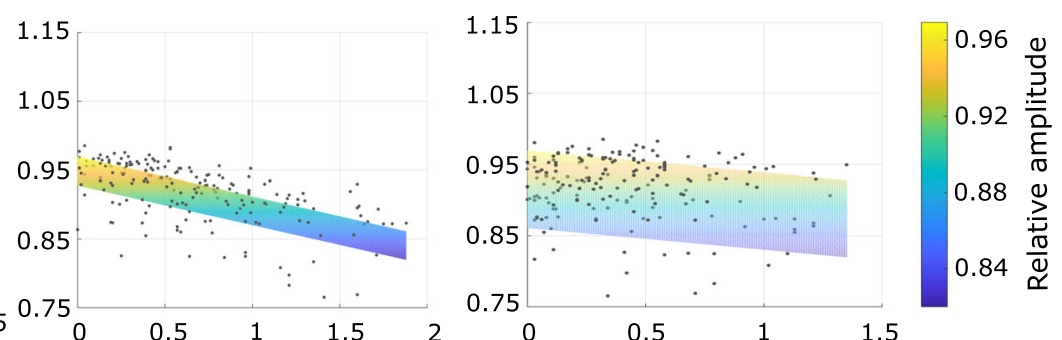

Relative prox-dist distance $(\mathrm{mm})$ Relative sept-temp distance $(\mathrm{mm})$

FIGURE 5 Legend on next page. 
of direction showed that most events propagated at speeds $<0.2 \mathrm{~mm} /$ $\mathrm{ms}$, and for $\geq 2 S D, 4-6 S D$, and $\geq 6 S D$ they were concentrated at directions closer to the proximodistal axis (Figure 7f, Supplementary Figures 12 and 13). Together, these results indicate that ripples either

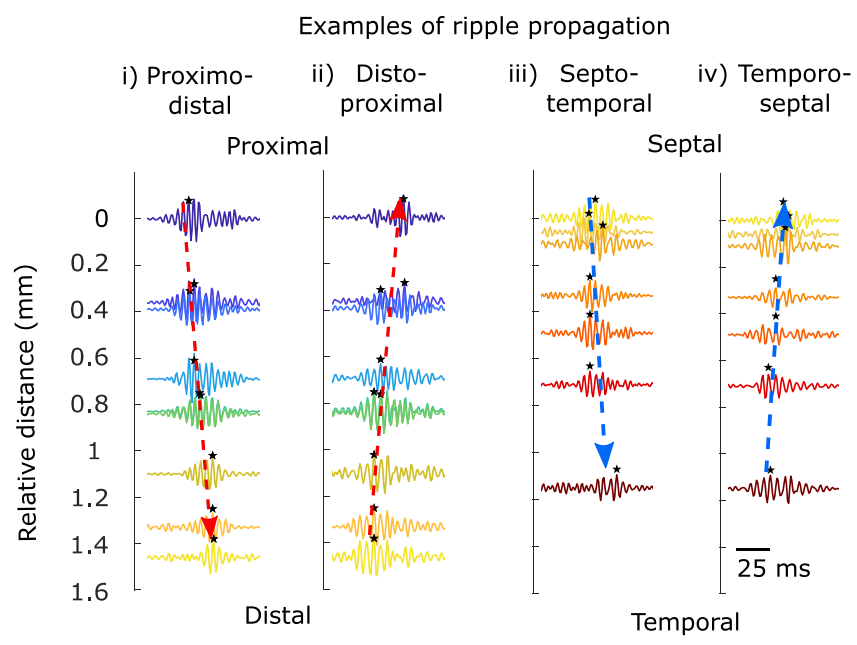

FIGURE 6 Examples of ripple propagation. Examples of ripples propagating from proximal to distal (rat 432) (i), distal to proximal (rat 432) (ii), septal to temporal (rat 392) (iii), and temporal to septal (rat 392) (iv). Arrangement of tetrodes is the same as in Figure 3. Dotted arrows indicate the direction of propagation [Color figure can be viewed at wileyonlinelibrary.com]

propagate along the proximodistal or septotemporal axis, and preferentially propagate along the proximodistal axis for events having higher amplitude ripples.

Next, we estimated average ripple propagation speeds. Events were classified based on the four directions of propagation (Figure 6) and analyzed separately. On average, each group showed a clear preference for the expected direction of propagation (Figure 8). For propagation along the proximodistal axis, the proximodistal partial slopes were significantly larger than the septotemporal partial slopes. For propagation along the septotemporal axis, the septotemporal partial slopes were significantly larger than the proximodistal partial slopes. The patterns remained the same for 2-4SD, 4-6 SD, and $\geq 6 S D$. For $\geq 2 S D$, ripples propagated at $0.081 \mathrm{~mm} / \mathrm{ms}$ in the proximodistal direction, $0.076 \mathrm{~mm} / \mathrm{ms}$ in the distoproximal direction, $0.063 \mathrm{~mm} / \mathrm{ms}$ in the septotemporal direction, and $0.056 \mathrm{~mm} / \mathrm{ms}$ in the temporoseptal direction (see Supplementary Figures 14-16 and Supplementary Table 15 for propagation speeds after subdividing propagating events by amplitude on the reference tetrode; see Supplementary Table 16 for ripple propagation statistics for individual rats).

The propagation statistics remained similar after controlling for differences in the spatial spreads of tetrodes across the proximodistal and septotemporal axes (Supplementary Figures 17-20 and Supplementary Table 17).

Having classified propagating ripples into the four directions of propagation, we looked at whether properties such as relative ripple amplitude, duration, frequency, and ripple bursts for ripples propagating along each of the four directions showed gradients along the proximodistal or septotemporal axis. Although we did see some significant gradients for relative ripple amplitude, duration, and frequency either along the proximodistal or septotemporal axis for the different directions of propagation in different amplitude groups, there were no patterns in gradients consistent with the patterns in the directions of propagation (Supplementary Figure 21-23 and Supplementary Table 18-20). The proportions of events having long-duration ripples and ripple bursts did not show any differences between proximodistal and distoproximal propagating ripples or between septotemporal and temporoseptal propagating ripples; however, the proportion of events with ripple bursts was nearly 10 times greater than those with longduration ripples (Supplementary Figure 24). We used a randomly selected matching number of nonpropagating ripples as a control for each direction of propagation. Similar to the propagating ripples, there were no patterns in significant gradients along the proximodistal and septotemporal axes (Supplementary Figure 21-23 and Supplementary Table 21-23). The proportions of events having long-duration ripples and ripple bursts also did not show any differences between proximodistal and distoproximal propagating ripples or between septotemporal and temporoseptal propagating ripples, but these proportions were 10 times smaller than those of the ripple bursts for propagating ripples (Supplementary Figure 24).

In summary, ripples propagated preferentially along the proximodistal axis, and propagating ripples showed a higher propensity to burst, regardless of the direction of propagation.

\section{4 | DISCUSSION}

In this study, we show that lower amplitude ripples preferentially occurred toward proximal CA1 while higher amplitude ripples preferentially occurred toward distal CA1. CA3 activity, which is known to induce sharp-wave ripples in CA1 (Buzsáki et al., 1992; Csicsvari, Hirase, Mamiya, \& Buzsáki, 2000), could give rise to the observed differences in the amplitude of ripples. Distal CA1 receives stronger projections from proximal CA3 while proximal CA1 receives stronger

FIGURE 5 Relative ripple amplitude. Figure organization is the same as Figure 4, but for relative ripple amplitude. Note the absence of gradients along the proximodistal and septotemporal axes in (b) and (c). (a) Proximodistal partial slope $=-0.025$ relative amplitude $/ \mathrm{mm}$, $p=2 \times 10^{-5 *}$; septotemporal partial slope $=-0.005$ relative amplitude $/ \mathrm{mm}, p=0.56$; one-tailed $t$-test for comparison of slopes along the two axes, $p=0.013^{*}$. See Supplementary Table 11 for statistics after subdividing ripples by amplitude on the reference tetrode and Supplementary Table 12 for relative ripple amplitude as a function of relative distance in individual rats [Color figure can be viewed at wileyonlinelibrary.com] 
(a) Polar distribution of propagation angles ( $\geq 2 \mathrm{SD}$ )

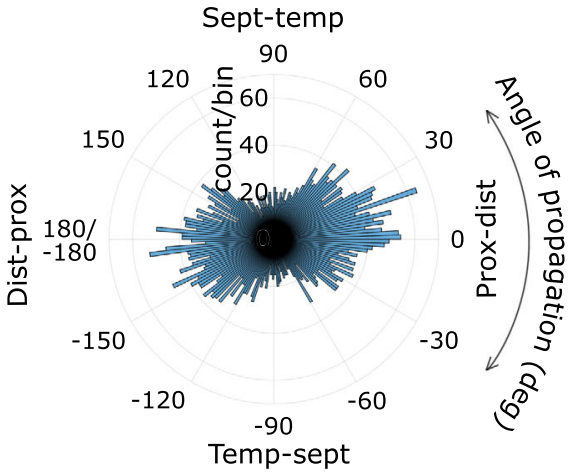

(b) Distribution of partial slopes

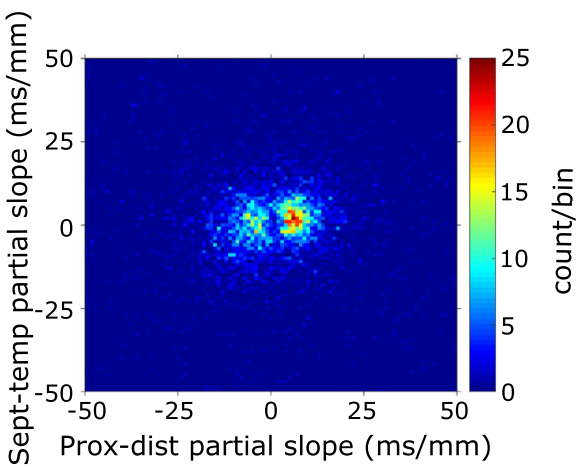

(c)

Polar distribution of propagation angles
i) 2-4 SD
ii) 4-6 SD

iii) $\geq 6 \mathrm{SD}$
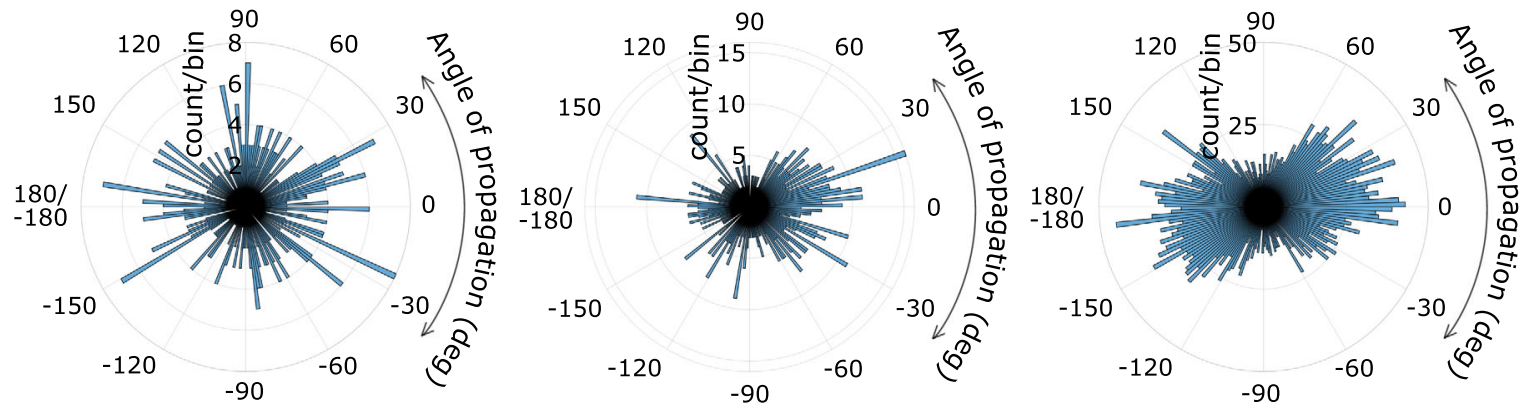

(d)

Distribution of partial slope p-values

(e) Distribution of $p$-values of $2 \mathrm{D}$ fit

ia) For proximo-distal partial slope

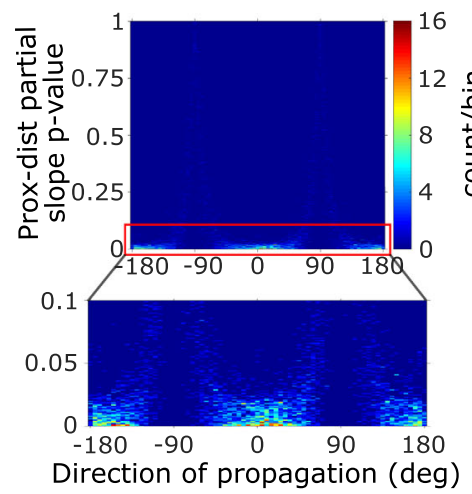

ib) For septo-temporal partial slope
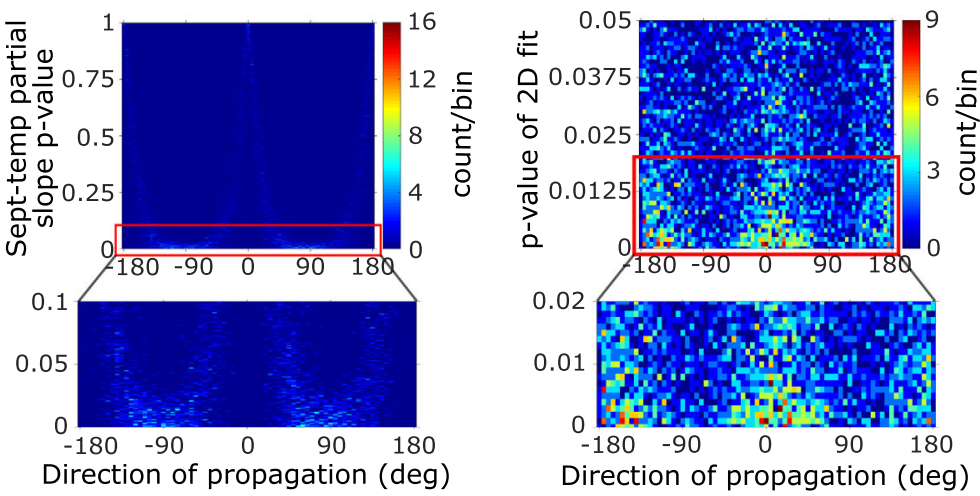

iia) For proximo-distal partial slope

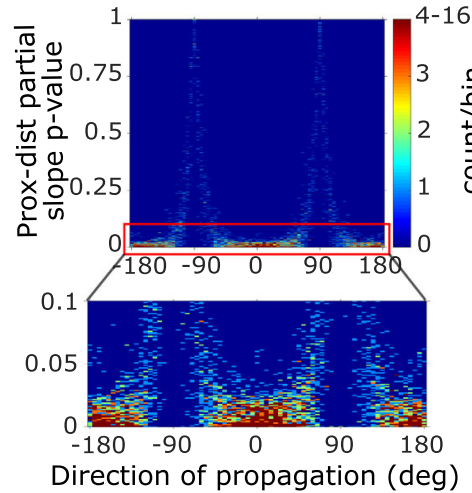

iib) For septo-temporal partial slope

(f) Distribution of resultant speeds
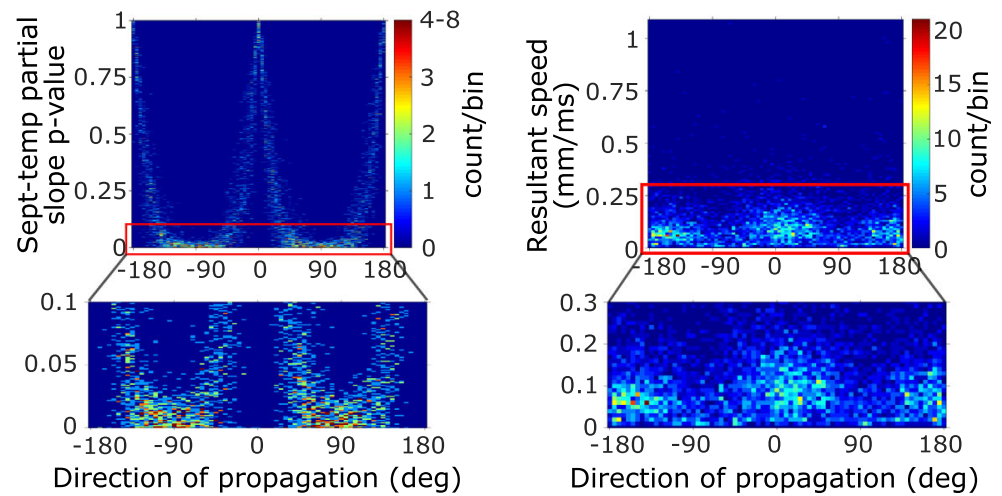

FIGURE 7 Legend on next page. 
projections from distal CA3 (Ishizuka, Weber, \& Amaral, 1990). Thus, the higher firing rates of proximal CA3 (Csicsvari et al., 2000) may induce higher amplitude ripples in distal CA1. We further showed that the amplitude and the probability of cooccurrence of ripples decreased as a function of distance from the reference. This fall-off was more reliable along the proximodistal axis than along a similar spatial extent of the septotemporal axis. The relatively small decline in amplitude and probability of cooccurrence across our spatial range of the septotemporal axis seem consistent with previous findings (Patel et al., 2013) within a similar spatial range. Additionally, in line with the Patel et al. (2013) study, we found that ripples propagate in CA1. They have shown that ripples propagate along a long range of the septotemporal axis. We showed that ripples propagate along the proximodistal and a comparable spatial extent of the septotemporal axis. Furthermore, the ripples preferentially propagated along the proximodistal axis (63\% of the propagating ripple events propagated along the proximodistal axis while $37 \%$ propagated along the septotemporal axis).

The propagation speeds along both the proximodistal $(\sim 0.08 \mathrm{~mm} /$ $\mathrm{ms})$ and septotemporal axes $(\sim 0.06 \mathrm{~mm} / \mathrm{ms})$ reported in this study are slower than the septotemporal speed reported in the Patel et al. (2013) study $(\sim 0.35 \mathrm{~mm} / \mathrm{ms})$. These differences could arise because of possible methodological differences in the experiment and analyses between the two studies. Patel et al. (2013) used only the ripple events during sleep, while our dataset includes periods of awake immobility as well as sleep, as we did not explicitly record the behavioral state of the animal while it rested on the pedestal. Unless we have only a small minority of propagating ripple events recorded during sleep in our dataset, the lack of fast propagating events (Supplementary Figure 25) is indicative of ripple propagation speeds being indeed slower in our dataset irrespective of the behavioral state of the rat. We could not determine the criteria used for identifying propagating ripples from the description in Patel et al. (2013), but they plot pairwise temporal differences for speed estimation. In contrast, our study uses the ripple timing over the entire tetrode distribution to both identify propagating ripples as well as measure propagation speeds. Differences in criteria used to classify events as propagating could influence the estimated speed.

Andersen, Bliss, and Skrede (1971) proposed that the hippocampal information processing is segregated in lamellae orthogonal to the long axis of the hippocampus (see Amaral \& Witter, 1989 for a thorough rebuttal of this influential hypothesis). While a cursory glance at our results may make one think they are in line with this lamellar hypothesis, they contradict it within the limits of the narrow septotemporal coverage in this study. The amplitude and cooccurrence gradients are stronger along the proximodistal (transverse) axis than along the septotemporal (long/longitudinal) axis. Segregation of information in transverse lamellae would predict a more rapid fall-off in amplitude/cooccurrence along the septotemporal axis than along the proximodistal axis. The ripples preferentially propagating along the proximodistal axis reported here only indicate that the information flows preferentially along this axis, without implications about whether or not the information is compartmentalized into lamellae. Only a study with electrodes spread over a larger extent of the septotemporal axis and the proximodistal axis can answer whether the ripples propagating along the proximodistal axis are confined to a narrow septotemporal section, or if there is a wave spread over a large extent of the septotemporal axis that propagates along the proximodistal axis. The $\sim 40 \%$ of ripples propagating along the short range of the septotemporal axis reported here and the septotemporal ripple propagation reported by Patel et al. (2013), however, directly contradict the lamellar segregation of information in the hippocampus.

What mechanism might give rise to the observed preference in propagation along the proximodistal axis versus the septotemporal axis? The two most obvious candidates are (a) the CA3 projections inducing sharp-wave ripple complexes in CA1 (Buzsáki et al., 1992; Csicsvari et al., 2000) and (b) the interactions of CA1 pyramidal cells with basket cells and bistratified cells, the interneurons implicated in ripple generation/timing within CA1 (Buzsáki, 2015; Csicsvari et al., 1999; Klausberger et al., 2004; Ylinen et al., 1995). The topographical organization of CA3 to CA1 connectivity is complex, both in terms of anatomy and physiology (Csicsvari et al., 2000; Ishizuka et al., 1990). Population bursts in CA3 correlated with CA1 sharpwaves show propagation from distal to proximal CA3 (Csicsvari et al., 2000), predicting a directional preference in ripple propagation, not seen in our data. It is possible that the higher firing rates of proximal CA3 pyramidal cells they report compensate by ensuring their postsynaptic targets reach ripple generation threshold sooner in the sharp-wave in some of the events. Whether the CA3 population bursts propagate more along the proximodistal axis than the septotemporal axis is unknown. Even if that were the case, the CA3

FIGURE 7 Ripple propagation. (a) Polar distribution of the angles of propagation for $\geq 2 \mathrm{SD}$. The following angles correspond to the given directions: proximodistal direction, $0^{\circ}$; distoproximal direction, $180^{\circ}$ or $-180^{\circ}$; septotemporal direction, $90^{\circ}$; temporoseptal direction, $-90^{\circ}$. Note the clear preference for the proximodistal axis over the septotemporal axis. (b) Distribution of proximodistal vs. septotemporal partial slopes obtained from multiple linear regression analysis of individual propagating events $(\geq 2 S D)$. Note the bimodal nature of the distribution of the proximodistal partial slopes but not the septotemporal partial slopes. (c) Polar distribution of the angles of propagation for 2-4 SD (i), 4-6 SD (ii), and $\geq 6 S D$ (iii). (d) Distributions of the $p$-values of partial slopes vs. direction (angle) of propagation for all propagating events ( $\geq 2$ SD). Panels (i) and (ii) are the same plots, however, the color scheme of (ii) has an upper limit of 4 (instead of the actual maximum bin count) to facilitate visualization of the pattern-note that the $p$-values are close to 0 about $0^{\circ},-180^{\circ}$, and $180^{\circ}$ and close to 1 about $-90^{\circ}$ and $90^{\circ}$ in (iia), and vice versa for the $p$-values in (iib). Each figure shows a zoomed in section below. (e) Distribution of the $p$-values of the multiple linear regression models vs. direction of propagation for all propagating events. ( $f$ ) Distribution of the resultant speed (obtained from vector analysis) vs. direction of propagation for all propagating events [Color figure can be viewed at wileyonlinelibrary.com] 
(a)

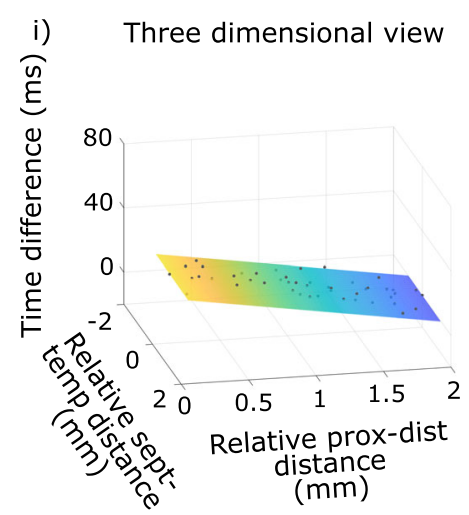

(b)

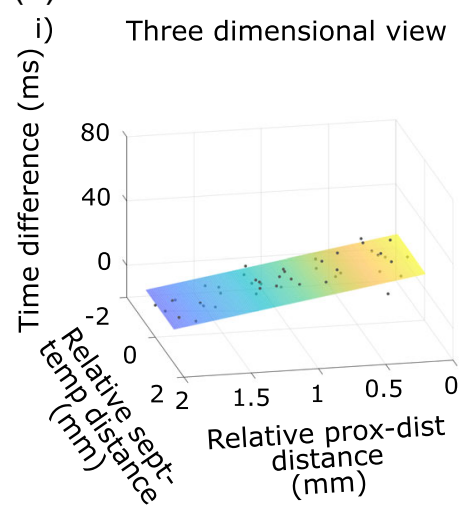

(c)

i) Three dimensional view

气ี

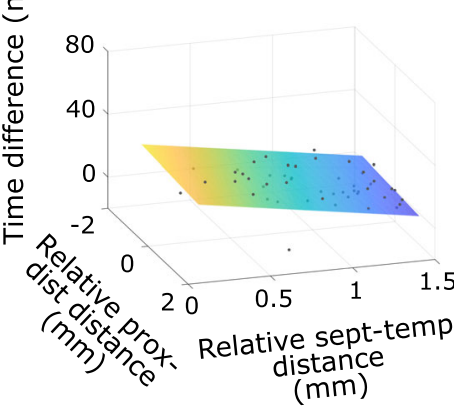

(d)

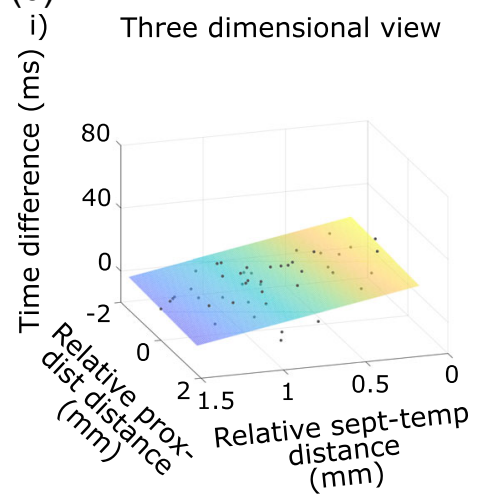
( $\mathrm{mm}$ )
Proximo-distal propagation

ii) Proximo-distal projection

iii) Septo-temporal projection
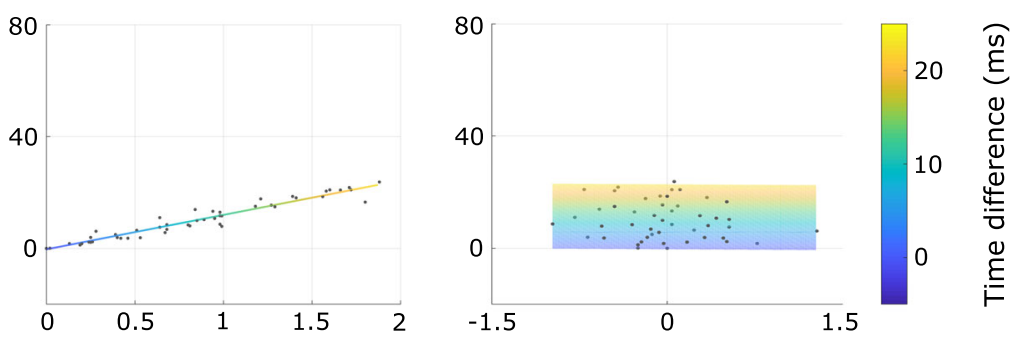

Relative prox-dist distance $(\mathrm{mm})$ Relative sept-temp distance $(\mathrm{mm})$
Disto-proximal propagation

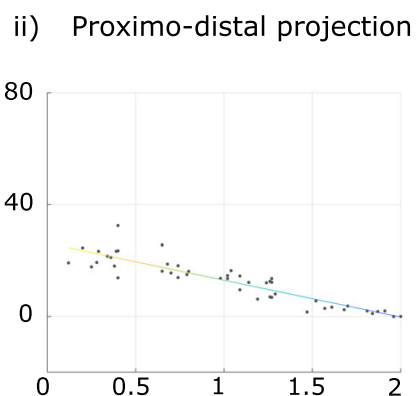

iii) Septo-temporal projection

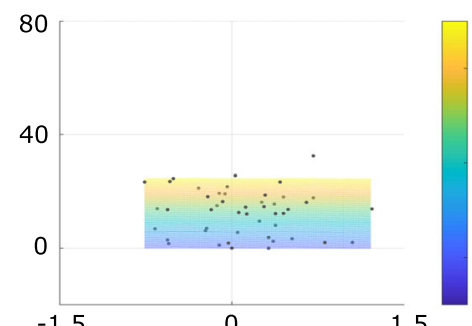

Relative prox-dist distance $(\mathrm{mm})$ Relative sept-temp distance $(\mathrm{mm})$

Septo-temporal propagation

ii) Proximo-distal projection

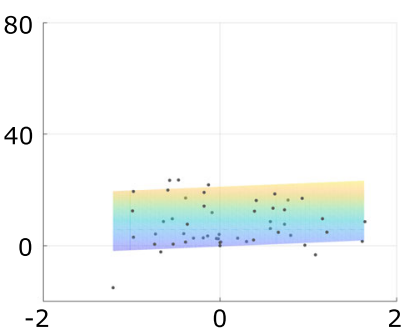

Relative prox-dist distance $(\mathrm{mm})$ Relative sept-temp distance $(\mathrm{mm})$

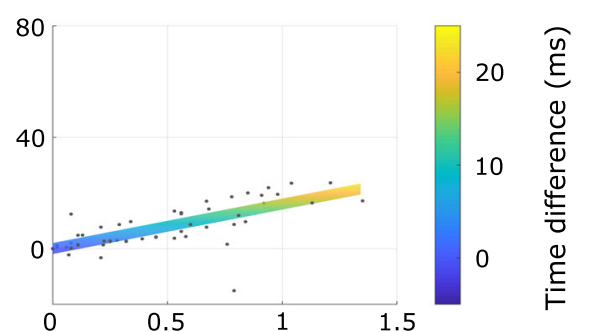

Temporo-septal propagation

ii) Proximo-distal projection

iii) Septo-temporal projection
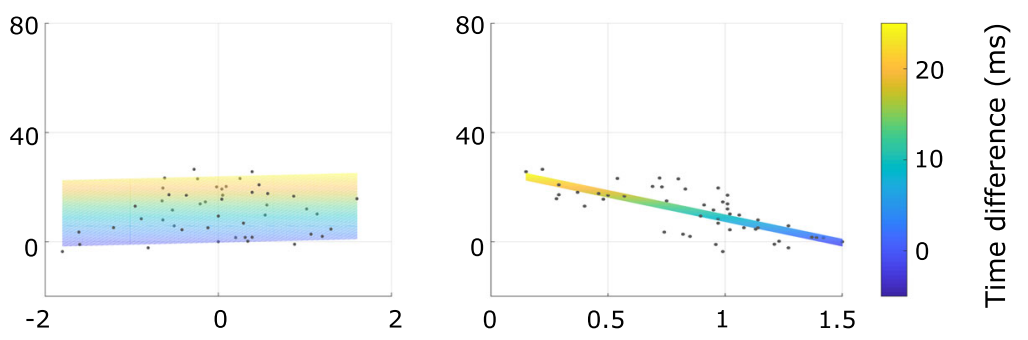

Relative prox-dist distance $(\mathrm{mm})$ Relative sept-temp distance $(\mathrm{mm})$

FIGURE 8 Legend on next page. 
to $\mathrm{CA} 1$ projection pattern is oblique, with proximal CA3 preferentially projecting more septally to distal CA1. Conversely, distal CA3 preferentially projects more temporally to proximal CA1 (Ishizuka et al., 1990). This CA3 to CA1 connectivity would predict an oblique spread of ripples within CA1 in response to CA3 activation propagating preferentially along the proximodistal axis, if CA3 to CA1 connectivity alone were driving CA1 ripple propagation. Basket cell and bistratified cell axonal distributions (Klausberger et al., 2004; Sik, Penttonen, Ylinen, \& Buzsáki, 1995) appear to be symmetric and show $\sim 1 \mathrm{~mm}$ spread in both proximodistal and septotemporal directions. Hence, the interneuron connectivity could possibly explain the nonpreferential propagation of the lower amplitude ripples. However, interneuron activity alone cannot account for the proximodistal vs. septotemporal difference in ripple propagation of higher amplitude ripples.

Even if the CA1 circuitry responsible for ripple generation is symmetric, asymmetric inputs can bias the starting point of propagating ripples to their target locations. Such asymmetry in inputs can lead to the observed anisotropy in preferred axes of ripple propagation. There are multiple potential candidates that may account for input asymmetry. The dentate gyrus (Sasaki et al., 2018; Sullivan et al., 2011), CA2 (Oliva, Fernández-Ruiz, Buzsáki, \& Berényi, 2016a), and GABAergic projections from the medial septum (Dragoi, Carpi, Recce, Csicsvari, \& Buzsáki, 1999; Viney et al., 2013) have been implicated in sharp-wave ripple initiation. Paucity of data about the projection patterns of these regions onto CA1 restricts our ability to understand their possible role in our observations.

LEC and MEC show differential projections to CA1 along the proximodistal axis (Naber et al., 2001; Steward \& Scoville, 1976). These differential projections could possibly explain the preferential propagation along the proximodistal axis over the septotemporal axis. There is some evidence for MEC activation during ripples. MEC shows high frequency events associated with ripples (Chrobak \& Buzsáki, 1996). Buzsáki (2015), using data from Mizuseki, Sirota, Pastalkova, and Buzsáki (2009), showed that superficial MEC activity peaks after the ripple peak, but the activity starts slowly ramping up some time before the peak (but see Chrobak \& Buzsáki, 1994; O'Neill, Boccara, Stella, Schoenenberger, \& Csicsvari, 2017). Sullivan et al. (2011) showed an increase in superficial MEC firing correlated with increased probability of ripples at the DOWN to UP state transition. MEC excitatory neurons show a strong activation preceding longer sharp-wave ripple complexes. These longer complexes appear to be two or more overlapping sharp-wave ripple complexes (Oliva, Fernández-Ruiz, Fermino de Oliveira, \& Buzsáki, 2018). Similarly, in mice, MEC LIII activity is essential for ripple bursts in CA1 during awake immobility (Yamamoto \& Tonegawa, 2017). We report here that ripple bursts on the reference tetrodes were 10 times as frequent during propagating ripples as during nonpropagating ripples, regardless of the direction of propagation. Given the role of MEC in driving ripple bursts (Oliva et al., 2018; Yamamoto \& Tonegawa, 2017), this suggests a possibility that there is an entorhinal drive during a substantial proportion of propagating ripples. To our knowledge, LEC activity during CA1 ripples has not been studied. If MEC and LEC are independently active during a subset of ripples, they may bias the ripples to start earlier in proximal or distal CA1 respectively, leading to the preferential propagation along the proximodistal axis observed in this study. Further studies are, however, required to ascertain the contribution of the various inputs described above to the propagation of CA1 ripples.

The biasing of a relatively large number of CA1 propagating ripples along the proximodistal axis could imply that transfer of information to the cortical areas for consolidation happens in an orderly fashion, with some events beginning with spatial (where) information and ending with sensory (what/landmark related spatial) information, while others beginning with sensory information and ending with spatial information (Deshmukh \& Knierim, 2011; Hafting et al., 2005; Hargreaves et al., 2005; Knierim et al., 2014). This biased CA1 ripple propagation would, in turn, add a layer of complexity to the reciprocal communication between the hippocampus and the cortex (Peyrache, Khamassi, Benchenane, Wiener, \& Battaglia, 2009; Rothschild, Eban, \& Frank, 2016).

In conclusion, our study shows a difference in ripple propagation along the proximodistal and the septotemporal axes, suggesting an anisotropy in the neural network along the two axes. Further studies are essential to discern the underlying mechanisms and functional significance of this observation.

\section{ACKNOWLEDGMENTS}

We thank Jeremy Johnson, Geeta Rao, Vyash Puliyadi, Amanda Smolinsky, Lou Blanpain, and Kimberley Christian for their support in

FIGURE 8 Ripple propagation speed for $\geq 2$ SD. (a-d) Distributions of relative time difference vs. relative distances along the proximodistal and septotemporal axes after classification of events into the four directions of propagation show clear narrow, linear trends along the expected direction of propagation. Column (i) shows a 3D (X-Y-Z) view, (ii) shows a proximodistal projection (X-Z view), and (iii) shows a septotemporal projection ( $\mathrm{Y}-\mathrm{Z}$ view) of column (i). Orientation of plots for propagation along the proximodistal axis (ai and bi) are different from those along the septotemporal axis (ci and di) for visualization purposes. (a) Proximodistal propagation: proximodistal partial slope $=12.3 \mathrm{~ms} / \mathrm{mm}, p<10^{-5 *}$; septotemporal partial slope $=0.22 \mathrm{~ms} / \mathrm{mm}, p=0.7$; one-tailed t-test for comparison of slopes along the two axes, $p<10^{-5 *}$. (b) Distoproximal propagation: proximodistal partial slope $=-13.2 \mathrm{~ms} / \mathrm{mm}, p<10^{-5 *}$; septotemporal partial slope $=-0.05 \mathrm{~ms} / \mathrm{mm}, p=0.96$; one-tailed t-test for comparison of slopes along the two axes, $p<10^{-5 *}$. (c) Septotemporal propagation: proximodistal partial slope $=1.33 \mathrm{~ms} / \mathrm{mm}, p=0.24$; septotemporal partial slope $=15.9 \mathrm{~ms} / \mathrm{mm}, p<10^{-5 *}$; one-tailed t-test for comparison of slopes along the two axes, $p<10^{-5 *}$. (d) Temporoseptal propagation: proximodistal partial slope $=0.77 \mathrm{~ms} / \mathrm{mm}, p=0.45$; septotemporal partial slope $=-17.9 \mathrm{~ms} / \mathrm{mm}, p<10^{-5 *}$; one-tailed t-test for comparison of slopes along the two axes, $p<10^{-5 *}$. See Supplementary Table 15 for statistics after subdividing ripples by amplitude and Supplementary Table 16 for statistics of ripple propagation in individual rats [Color figure can be viewed at wileyonlinelibrary.com] 
data collection; Aditi Bishnoi, Indraja Jakhalekar and Rajat Saxena for their inputs on the manuscript; Daniel Levenstein for the wavelet transform code. This work was supported by Wellcome Trust/DBT India Alliance Grant IA/S/13/2/501024. Data collection was supported by R01 NS039456 (J. Knierim, PI).

\section{AUTHOR CONTRIBUTIONS}

Mekhala Kumar conceived the project, analyzed the data, and wrote the manuscript. Sachin S. Deshmukh collected the data, conceived the project, and wrote the manuscript.

\section{DATA AVAILABILITY STATEMENT}

The data that support the findings of this study are available from the corresponding author upon reasonable request.

\section{ORCID}

Mekhala Kumar (DD https://orcid.org/0000-0002-9395-0376

Sachin S. Deshmukh (DD https://orcid.org/0000-0002-8421-4158

\section{REFERENCES}

Amaral, D. G., \& Witter, M. P. (1989). The three-dimensional organization of the hippocampal formation: A review of anatomical data. Neuroscience, 31, 571-591.

Andersen, P., Bliss, V. P., \& Skrede, K. K. (1971). Lamellar organization of hippocampal excitatory pathways. Experimental Brain Research, 13, 222-238.

Buzsáki, G. (2015). Hippocampal sharp wave-ripple: A cognitive biomarker for episodic memory and planning. Hippocampus, 25, 1073-1188.

Buzsáki, G., Horváth, Z., Urioste, R., Hetke, J., \& Wise, K. (1992). Highfrequency network oscillation in the hippocampus. Science, 256, 1025-1027.

Chrobak, J. J., \& Buzsáki, G. (1994). Selective activation of deep layer (V-VI) Retrohippocampal cortical neurons during hippocampal sharp waves in the behaving rat. The Journal of Neuroscience, 14, 6160-6170.

Chrobak, J. J., \& Buzsáki, G. (1996). High-frequency oscillations in the output networks of the hippocampal-Entorhinal Axis of the freely behaving rat. The Journal of Neuroscience, 16, 3056-3066.

Csicsvari, J., Hirase, H., Czurkó, A., Mamiya, A., \& Buzsáki, G. (1999). Oscillatory coupling of hippocampal pyramidal cells and interneurons in the behaving rat. The Journal of Neuroscience, 19, 274-287.

Csicsvari, J., Hirase, H., Mamiya, A., \& Buzsáki, G. (2000). Ensemble patterns of hippocampal CA3-CA1 neurons during sharp wave - Associated population events. Neuron, 28, 585-594.

Deshmukh, S. S., \& Knierim, J. J. (2011). Representation of non-spatial and spatial information in the lateral entorhinal cortex. Frontiers in Behavioral Neuroscience, 5, 1-13.

Dragoi, G., Carpi, D., Recce, M., Csicsvari, J., \& Buzsáki, G. (1999). Interactions between hippocampus and medial septum during sharp waves and theta oscillation in the behaving rat. The Journal of Neuroscience, 19, 6191-6199.

Hafting, T., Fyhn, M., Molden, S., Moser, M. B., \& Moser, E. I. (2005). Microstructure of a spatial map in the entorhinal cortex. Nature, 436, 801-806.

Hargreaves, E. L., Rao, G., \& Knierim, J. J. (2005). Major dissociation between medial and lateral entorhinal input to dorsal hippocampus. Science, 308, 1792-1794.

Henriksen, E. J., Colgin, L. L., Barnes, C. A., Witter, M. P., Moser, M. B., \& Moser, E. I. (2010). Spatial representation along the Proximodistal Axis of CA1. Neuron, 68, 127-137.

Ishizuka, N., Weber, J., \& Amaral, D. G. (1990). Organization of intrahippocampal projections originating from CA3 pyramidal cells in the rat. The Journal of Comparative Neurology, 295, 580-623.
Jung, M. W., Wiener, S. I., \& McNaughton, B. L. (1994). Comparison of spatial firing characteristics of units in dorsal and ventral hippocampus of the rat. The Journal of Neuroscience, 14, 7347-7356.

Klausberger, T., Márton, L. F., Baude, A., Roberts, J. D. B., Magill, P. J., \& Somogyi, P. (2004). Spike timing of dendrite-targeting bistratified cells during hippocampal network oscillations in vivo. Nature Neuroscience, 7, 41-47.

Knierim, J. J. (2002). Dynamic interactions between local surface cues, distal landmarks, and intrinsic circuitry in hippocampal place cells. The Journal of Neuroscience, 22, 6254-6264.

Knierim, J. J., Neunuebel, J. P., \& Deshmukh, S. S. (2014). Functional correlates of the lateral and medial entorhinal cortex: Objects, path integration, and local-global reference frames. Philosophical Transactions of the Royal Society of London. Series B, Biological Sciences, 369, 20130369.

Maurer, A. P., VanRhoads, S. R., Sutherland, G. R., Lipa, P., \& McNaughton, B. L. (2005). Self-motion and the origin of differential spatial scaling along the septo-temporal axis of the hippocampus. Hippocampus, 15, 841-852.

Mizuseki, K., Diba, K., Pastalkova, E., \& Buzsáki, G. (2011). Hippocampal CA1 pyramidal cells form functionally distinct sublayers. Nature Neuroscience, 14, 1174-1181.

Mizuseki, K., Sirota, A., Pastalkova, E., \& Buzsáki, G. (2009). Theta oscillations provide temporal windows for local circuit computation in the entorhinal-hippocampal loop. Neuron, 64, 267-280.

Naber, P. A., Lopes da Silva, F. H., \& Witter, M. P. (2001). Reciprocal connections between the entorhinal cortex and hippocampal fields CA1 and the subiculum are in register with the projections from CA1 to the subiculum. Hippocampus, 11, 99-104.

Oliva, A., Fernández-Ruiz, A., Buzsáki, G., \& Berényi, A. (2016a). Role of hippocampal CA2 region in triggering sharp-wave ripples. Neuron, 91 , 1342-1355.

Oliva, A., Fernández-Ruiz, A., Buzsáki, G., \& Berényi, A. (2016b). Spatial coding and physiological properties of hippocampal neurons in the Cornu Ammonis subregions. Hippocampus, 26, 1593-1607.

Oliva, A., Fernández-Ruiz, A., Fermino de Oliveira, E., \& Buzsáki, G. (2018). Origin of gamma frequency power during hippocampal sharp-wave ripples. Cell Reports, 25, 1693-1700.

O'Neill, J., Boccara, C. N., Stella, F., Schoenenberger, P., \& Csicsvari, J. (2017). Superficial layers of the medial entorhinal cortex replay independently of the hippocampus. Science, 355, 184-188.

Patel, J., Schomburg, E. W., Berényi, A., Fujisawa, S., \& Buzsáki, G. (2013). Local generation and propagation of ripples along the Septotemporal Axis of the hippocampus. The Journal of Neuroscience, 33, 17029-17041.

Paxinos, G., \& Watson, C. (2007). The rat brain in stereotaxic coordinates (6th ed.). Burlington, MA: Elsevier/Academic Press.

Peyrache, A., Khamassi, M., Benchenane, K., Wiener, S. I., \& Battaglia, F. P. (2009). Replay of rule-learning related neural patterns in the prefrontal cortex during sleep. Nature Neuroscience, 12, 919-926.

Rothschild, G., Eban, E., \& Frank, L. M. (2016). A Cortio-hippocampal cortical loop of information processing during memory consolidation. Nature Neuroscience, 20, 251-259.

Sasaki, T., Piatti, V. C., Hwaun, E., Ahmadi, S., Lisman, J. E., Leutgeb, S., \& Leutgeb, J. K. (2018). Dentate network activity is necessary for spatial working memory by supporting CA3 sharp-wave ripple generation and prospective firing of CA3 neurons. Nature Neuroscience, 21, 258-269.

Sik, A., Penttonen, M., Ylinen, A., \& Buzsáki, G. (1995). Hippocampal CA1 interneurons: An in vivo intracellular labelling study. The Journal of Neuroscience, 15, 6651-6665.

Stensola, H., Stensola, T., Solstad, T., Frøland, K., Moser, M. B., \& Moser, E. I. (2012). The entorhinal grid map is discretized. Nature, 492, 72-78.

Steward, O., \& Scoville, S. A. (1976). Cells of origin of entorhinal cortical afferents to the hippocampus and fascia dentata of the rat. The Journal of Comparative Neurology, 169, 347-370. 
Sullivan, D., Csicsvari, J., Mizuseki, K., Montgomery, S., Diba, K., \& Buzsáki, G. (2011). Relationships between hippocampal sharp waves, ripples, and fast gamma oscillation: Influence of dentate and entorhinal cortical activity. The Journal of Neuroscience, 31, 8605-8616.

Viney, T. J., Lasztoczi, B., Katona, L., Crump, M. G., Tukker, J. J., Klausberger, T., \& Somogyi, P. (2013). Network state-dependent inhibition of identified hippocampal CA3 axo-axonic cells in vivo. Nature Neuroscience, 16, 1802-1811.

Witter, M. P., \& Amaral, D. G. (2004). Hippocampal formation. In G. Paxinos(Ed.), The rat nervous system (pp. 637-704). Amsterdam: Elsevier/Academic Press.

Yamamoto, J., \& Tonegawa, S. (2017). Direct medial Entorhinal cortex input to hippocampal CA1 is crucial for extended quiet awake replay. Neuron, 96, 217-227.

Ylinen, A., Bragin, A., Nádasdy, Z., Jandó, G., Szabó, I., Sik, A., \& Buzsáki, G. (1995). Sharp wave-associatedhigh-frequency oscillation $(200 \mathrm{~Hz})$ in the intact hippocampus: Network and intracellular mechanisms. The Journal of Neuroscience, 15, 30-46.

\section{SUPPORTING INFORMATION}

Additional supporting information may be found online in the Supporting Information section at the end of this article.

How to cite this article: Kumar M, Deshmukh SS. Differential propagation of ripples along the proximodistal and septotemporal axes of dorsal CA1 of rats. Hippocampus. 2020;

1-17. https://doi.org/10.1002/hipo.23211 